\title{
Composition of the fish fauna in a tropical estuary: the ecological guild approach
}

\author{
Valdimere Ferreira ${ }^{1}$, François Le Loc'h ${ }^{2}$, Frédéric Ménard ${ }^{3}$, Thierry Frédou ${ }^{1}$, \\ Flávia L. Frédou ${ }^{1}$ \\ ${ }^{1}$ Universidade Federal Rural de Pernambuco (UFRPE), Rua Dom Manuel de Medeiros, s/n, 52171-900, Recife, Brazil. \\ (VF) (Corresponding author) E-mail: valdimereferreira@yahoo.com.br. ORCID iD: https://orcid.org/0000-0002-5051-9439 \\ (TF) E-mail: thierry.fredou@ufrpe.br. ORCID iD: https://orcid.org/0000-0002-0510-6424 \\ (FLF) E-mail: flavia.fredou@ufrpe.br. ORCID iD: https://orcid.org/0000-0001-5492-7205 \\ 2 IRD, Univ. Brest, CNRS, Ifremer, UMR LEMAR, F-29280 Plouzané, France. \\ (FLL) E-mail: francois.le.loch@ird.fr. ORCID iD: https://orcid.org/0000-0002-3372-6997 \\ ${ }^{3}$ Aix Marseille Univ., Université de Toulon, CNRS, IRD, UMR MIO, Marseille, France. \\ (FM) E-mail: frederic.menard@ird.fr. ORCID iD: https://orcid.org/0000-0003-1162-660X
}

\begin{abstract}
Summary: Ecological guilds have been widely applied for understanding the structure and functioning of aquatic ecosystems. This study describes the composition and the spatio-temporal changes in the structure of the fish fauna and the movements between the estuary and the coast of a tropical estuary, the Itapissuma/Itamaracá Complex (IIC) in northeastern Brazil. Fish specimens were collected during the dry and rainy seasons in 2013 and 2014. A total of 141 species of 34 families were recorded. Almost half of the species (66 species, $47 \%)$ were exclusive to the estuary and 50 species $(35 \%)$ to the coast; 25 $(18 \%)$ were common to both environments. Marine species were dominant in both richness and biomass as they explore the environment during part of their life cycle, whereas estuarine species were dominant in abundance. Marine stragglers displayed a higher richness, abundance and biomass in the coastal waters. The estuarine environment was dominated by zoobenthivores in terms of richness, while detritivores prevailed in abundance and biomass. Zoobenthivores had the highest richness and abundance in coastal waters, while piscivores had the highest biomass. The IIC supports a rich fauna with a diverse trophic structure and is an important feeding and development area for migratory species.
\end{abstract}

Keywords: fish; functional attribute; habitat; Pernambuco; spatial-temporal distribution.

Composición de la fauna de peces de un estuario tropical: el enfoque del grupo ecológico

Resumen: Los grupos ecológicos se han aplicado ampliamente para comprender la estructura y el funcionamiento de los ecosistemas acuáticos. Este estudio describe la composición y los cambios espaciotemporales en la estructura de la fauna de peces y los movimientos entre el estuario y la costa de un estuario tropical (Complejo Itapissuma/Itamaracá - CII) en el noreste de Brasil. Los especímenes de peces fueron recolectados durante la estación seca y lluviosa del 2013 y 2014 . Se registraron un total de 141 especies de 34 familias. Casi la mitad de las especies (66 especies, $47 \%$ ) eran exclusivas del estuario y 50 especies $(35 \%)$ de la costa; 25 (18\%) fueron comunes a ambos ambientes. Las especies marinas fueron dominantes tanto en riqueza como en biomasa, ya que exploraron el medio ambiente durante parte de su ciclo de vida, mientras que las especies estuarinas dominaron considerando la abundancia. Las especies marino-dependientes mostraron una mayor riqueza, abundancia y biomasa en las aguas costeras. El ambiente estuarino fue dominado por zoobentívoros en términos de riqueza, mientras que los detritívoros prevalecieron en abundancia y biomasa. Los zoobentívoros tuvieron la mayor riqueza y abundancia en las aguas costeras, mientras que los piscívoros mostraron la mayor biomasa. El CII sostiene una rica fauna con una estructura trófica diversa y es un área relevante de alimentación y desarrollo para las especies migratorias.

Palabras clave: peces; atributo funcional; hábitat; Pernambuco; distribución espaciotemporal.

Citation/Como citar este artículo: Ferreira V., Le Loc'h F., Ménard F., Frédou T., Frédou F.L. 2019. Composition of the fish fauna in a tropical estuary: the ecological guild approach. Sci. Mar. 83(2): 133-142. https://doi.org/10.3989/ scimar.04855.25A

Editor: E. Macpherson.

Received: September 10, 2018. Accepted: March 14, 2019. Published: May 15, 2019.

Copyright: (C) 2019 CSIC. This is an open-access article distributed under the terms of the Creative Commons Attribution 4.0 International (CC BY 4.0) License. 


\section{INTRODUCTION}

The ichthyofauna can be described and classified through the functional attributes of organisms, mainly based on the trophic level, reproductive strategy or use of the environment (Elliott et al. 2007). The functional attributes divide the species into guilds, defined as groups of species that exploit the same class of environmental resources in a similar way (Root 1967). The guild approach allows a better understanding of the ecology and role of the biota in the ecosystem (Elliott et al. 2007). It may help identify overexploited resources through changes in the composition of the food web (Garrison and Link 2000) and of the energy flows in the system (Harrison and Whitfield 2008). The guild approach also helps to understand the effects of climate changes on the structure and composition of fish fauna (Feyrer et al. 2015).

Trophic and estuarine use guilds have been widely applied to understand the structure and functioning of aquatic ecosystems, the movement pattern between environments and their use as feeding, breeding or development grounds (Elliott et al. 2007). Estuarine use guilds reflect migratory patterns and physiological adaptations of species that explore the area throughout their life cycle or part of it (Elliott et al. 2007). Trophic guilds are useful in the comprehension of the feeding habits of a species (Elliott et al. 2007). Its ecological relationships and the energy flows (Paiva et al. 2008) may reflect the possible strategies for avoiding competition or for optimizing the consumption of available resources (Angel and Ojeda 2001).

Estuaries are important transitional environments for the movement of the ichthyofauna between the continental basins and the ocean (Ray 2005). As an ecotone, estuaries link marine and freshwater ecosystems (Gray and Elliott 2009), and persistent environmental fluctuations place considerable physiological demands on the species inhabiting the area (Elliott and Quintino 2007). Many species are dependent on estuarine environments; several marine species are considered visitors and explore estuarine habitats during their ontogenetic development, evidencing the relationship with coastal environments (Able 2005). Therefore, defining the relationships between species and their functional roles within communities is critical for understanding the dynamics of the ecosystem and fundamental for the implementation of ecosystem-based fisheries management (Buchheister and Latour 2015).

The Brazilian coast hosts large estuarine complexes along the $187 \mathrm{~km}$ of the coast of Pernambuco, and several areas are considered of great environmental importance (CPRH 2010). The variety of habitats, along with the complexity of interactions within the fish community and the migratory nature of many species, hampers the assessment of the overall condition of the area (Vasconcelos Filho et al. 2003).

Using the ecological guilds approach, this study describes the composition and structure of the fish fauna along a tropical estuarine complex in order to identify and explain the main patterns of seasonal and spatial variations in assemblage composition. The study also discusses the importance of the use of the ecological guilds approach to assess the effects of multiple anthropogenic pressures on the structure and functioning of fish communities in tropical estuaries.

\section{MATERIALS AND METHODS}

\section{Study area}

The Itapissuma/Itamaracá Complex (IIC), located in Pernambuco, northeastern Brazil, within the Santa Cruz Environmental Preservation Area (APA Santa Cruz), is considered highly productive (Macêdo et al. 2000), hosting the largest fishery port in the state. Fishery is a very important socio-economical activity in the IIC, generating income and proteins for the local communities (CPRH 2010). Conversely, this ecosystem is exposed to multiple pressures from industrial pollution, domestic sewage discharge, urban expansion, land reclamation and fisheries (Medeiros et al. 2001). In addition, it has a large variety of connecting habitats favouring the development of the ichtyofauna (Vasconcelos Filho et al. 2009). The IIC is composed of the estuarine area, the Santa Cruz Channel and the adjacent sea, locally named the "Inner Sea" (Fig. 1). The Santa Cruz channel has a length of $22 \mathrm{~km}$, a width ranging from 0.6 to $1.5 \mathrm{~km}$ and a depth ranging from 2 to $5 \mathrm{~m}$ in the central part of the channel, reaching $10 \mathrm{~m}$ at the northern and southern bars that connect the channel to the sea (Vasconcelos Filho and Oliveira 1999). The channel bottom consists of quartz sand banks and dark, reductive and dense mud patches. The muddy banks are dominated by Rhyzophora mangle, Laguncularia racemosa, Avicennia sp. and Conocarpus erectus, and by meadows of the marine phanerogam, Halodule wrightii. Surface water temperature varies between $25^{\circ} \mathrm{C}$ and $31^{\circ} \mathrm{C}$ and salinity between 18 and 34. The Inner Sea, corresponding to the coastal area hereafter, with a depth of 2 to $5 \mathrm{~m}$, is characterized by a reef barrier parallel to the coast, located $4 \mathrm{~km}$ from the beach (Kempf 1970), which functions as a barrier between nearshore and shelf waters. The substrate is formed by terrigenous sediments from the mouth of the Jaguaribe River and the Santa Cruz Channel, and carbonates from the reef barrier (Almeida and Manso 2011), partially covered by large banks of phanerogams (Kempf 1970). The carbonaceous material is the result of the decomposition of rocks and quartz, sand, mollusc shells, foraminifera and calcareous algal fragments. In the Inner Sea, water temperature varies between $27^{\circ} \mathrm{C}$ and $30.8^{\circ} \mathrm{C}$ and the average annual salinity is 34 .

\section{Data collection}

Fish specimens were collected during the dry season (January, February, March, November) and the rainy season (May, July, August) in 2013 and 2014 in the Santa Cruz Channel and the Inner Sea. In order to minimize biases due to gear selectivity, different fishing gears were combined for accessing and sampling different habitats and maximizing the collection of fish individuals (Table S1, Supplementary material). In the estuary, three 25 -minute sets with a seine net and one 


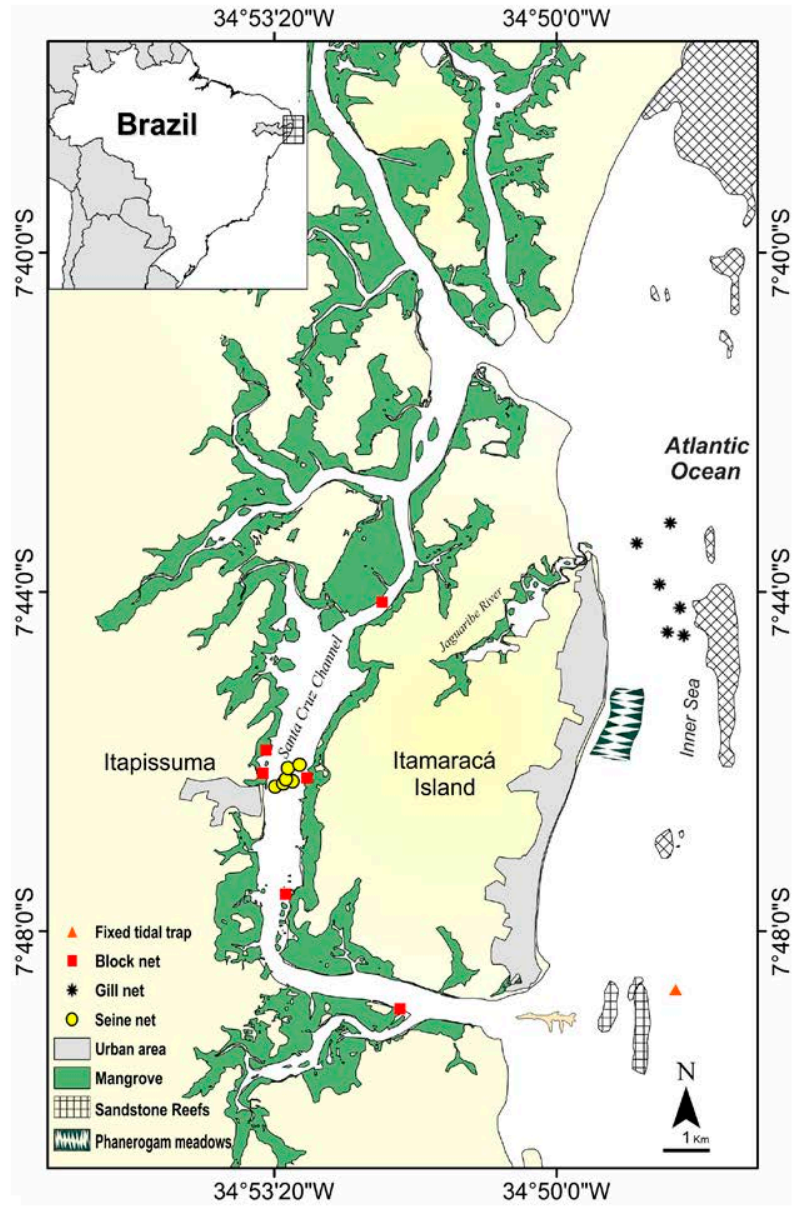

Fig. 1. - The study area of the Itapissuma/Itamaracá Complex, Pernambuco, Brazil and location of fish sampling points.

6-hour set with a block net were carried out quarterly. The seine net was $67.5 \mathrm{~m}$ long and had a mesh size of $10 \mathrm{~mm}$. The block net was $348 \mathrm{~m}$ long and had a mesh size of 60,70 and $80 \mathrm{~mm}$. On the coast, samples were obtained quarterly with a gillnet ( 3 sets of two hours each) and with a fixed tidal trap (6 fishing days). The gill net had mesh sizes of 50,70 and $80 \mathrm{~mm}$, and was $690 \mathrm{~m}$ long, and the fixed tidal trap had a diameter of $27 \mathrm{~m}$ and a mesh size of $70 \mathrm{~mm}$.

In the field, the fish fauna was conserved in thermal boxes with ice, and samples were frozen in the laboratory to be identified. Taxonomic classification followed Nelson et al. (2016).

\section{Data analysis}

Firstly, we computed a species accumulation curve with the non-parametric Bootstrap method (Smith and van Belle 1984) to assess whether the fish community was exhaustively sampled. This method assumes that all species occur randomly without taking into account species abundance, i.e. the method does not distinguish rare and abundant species (Smith and van Belle 1984). The index and standard deviations of the estimates were obtained through the analytical equation of Colwell et al. (2004) using the EstimateS software v. 9 9.1.0 (Colwell 2013).
The composition of the fish fauna was reported in terms of absolute species richness (S) and, for each species, frequency of occurrence $(\% \mathrm{FO})$ and relative abundance in number $(\% \mathrm{~N})$ and biomass $(\% \mathrm{~B})$. Species were considered to be abundant according to the Garcia and Vieira (2001) classification when $\% \mathrm{~N}$ was greater than $100 / \mathrm{S}$, where $\mathrm{S}$ is the number of species recorded in the area. A species was defined as frequent when its \%FO value for a given area was greater than $50 \%$. The combination of these parameters allowed the species to be classified into four categories: abundant and frequent $(\% \mathrm{~N}>100 / \mathrm{S}$ and $\% \mathrm{FO} \geq 50 \%)$; abundant but infrequent $(\% \mathrm{~N}>100 / \mathrm{S}$ and $\% \mathrm{FO}<50 \%)$; less abundant but frequent $(\% \mathrm{~N}<100 / \mathrm{S}$ and $\% \mathrm{FO} \geq 50 \%$ ) and less abundant and infrequent $(\% \mathrm{~N}<100 / \mathrm{S}$ and $\% \mathrm{FO}<50 \%)$.

Each species was assigned to an estuarine use functional group: marine stragglers, marine migrants and estuarine species, according to the classification proposed by Elliott et al. (2007). This classification is based on the type, frequency and period of use of the estuarine environment, and the abundance of the species in the estuary. In addition, each species was assigned to a trophic functional group based on local information about feeding preferences and strategies, according to the categories proposed by Elliott et al. (2007). The trophic functional groups were zooplanktivores, detritivores, piscivores, zoobenthivores, herbivores and omnivores. Information on trophic guilds were obtained in studies carried out in the IIC, in the scientific literature or, when not available, based on the WoRMS Editorial Board (2019) and FishBase project (Froese and Pauly 2007) (Table S2, Supplementary material). For each environment (estuary and coast) and season (dry and rainy), the estuarine use guild and the trophic guild were reported in terms of richness $(\% \mathrm{~S})$, abundance $(\% \mathrm{~N})$ and biomass $(\% \mathrm{~B})$.

We computed multivariate analyses to investigate the spatial and temporal variations in the structure of the fish community, considering the absolute richness of estuarine use guild and the richness of trophic guild by environment and by season. To analyse the guild composition, a principal coordinate analysis (PCO) based on Bray-Curtis distances was applied. The differences of the contribution of guilds between environments and seasons was tested by permutational multivariate analysis (PERMANOVA) (Anderson 2001) performed with a Bray-Curtis distance matrix built on square-root-transformed data. Multivariate analyses were performed with the $\mathrm{R}$ software (R Core Team 2018).

\section{RESULTS}

\section{Fish assemblage}

A total of 140 species (135 Actinopterygii and 5 Elasmobranchii) of 34 families were recorded in the IIC (Table 1). For both coastal and estuarine areas, the species accumulation curve did not stabilize towards asymptotic values (Fig. S1, Supplementary material). However, a large portion of the estimated richness was 
Table 1. - Composition of the ichthyofauna captured in the Itapissuma/Itamaracá Complex. D, dry; R, rainy; EUFG, estuarine use functional group; ES, estuarine species; MM, marine migrants; MS, marine stragglers; FMFG, feeding mode functional group; HV, herbivore; DV, detritivore; OV, omnivore; PV, piscivore; ZB, zoobenthivore; ZP, zooplanktivore; E, estuary; C, coast; N, abundance; B, biomass; FO, occurrence frequency; E, estuary; C, coast; IR, relative importance: 1, abundant and frequent; 2 , abundant and infrequent; 4, less abundant and infrequent; $(*)$ Species present in all the studied environments. Sea $=$ Season. $* *$ biomass $(\%)<0.01$

\begin{tabular}{|c|c|c|c|c|c|c|c|c|c|c|c|}
\hline \multirow{2}{*}{ Species } & \multirow{2}{*}{ Sea } & \multirow{2}{*}{\multicolumn{2}{|c|}{ EUFG FMFG }} & \multicolumn{2}{|c|}{$\mathrm{N}(\%)$} & \multicolumn{2}{|c|}{$\mathrm{B}(\%)$} & \multicolumn{2}{|c|}{$\mathrm{FO}(\%)$} & & \\
\hline & & & & $\mathrm{E}$ & $\mathrm{C}$ & $\mathrm{E}$ & $\mathrm{C}$ & $\mathrm{E}$ & $\mathrm{C}$ & $\mathrm{E}$ & $\mathrm{C}$ \\
\hline Carcharhinidae & & & & & & & & & & & \\
\hline Rhizoprionodon porosus (Poey, 1861) & $\mathrm{D}$ & MS & PV & & 0.11 & & 0.06 & & 1.9 & & 4 \\
\hline Rhizoprionodon lalandii (Valenciennes, 1839) & $\mathrm{D}$ & MS & PV & & 0.11 & & 0.04 & & 1.9 & & 4 \\
\hline Dasyatidae & & & & & & & & & & & \\
\hline Hypanus guttatus (Bloch and Schneider, 1801) * & $\mathrm{D} / \mathrm{R}$ & MS & ZB & 0.01 & 0.11 & 0.07 & 2.57 & 3.4 & 1.9 & 4 & 4 \\
\hline Hypanus marianae Gomes Rosa and Gadig, 2000 & $\mathrm{D} / \mathrm{R}$ & MS & $\mathrm{ZB}$ & & 0.22 & & 0.14 & & 3.8 & & 4 \\
\hline Elopidae & & & & & & & & & & & \\
\hline Elops saurus (Linnaeus, 1766) & $\mathrm{D}$ & MS & PV & 0.01 & & 0.07 & & 3.4 & & 4 & \\
\hline Muraenidae & & & & & & & & & & & \\
\hline Gymnothorax funebris Ranzani, 1839 & $\mathrm{R}$ & MS & $\mathrm{ZB}$ & & 0.43 & & 1.53 & & 5.8 & & 4 \\
\hline Gymnothorax ocellatus Agassiz, 1831* & $\mathrm{D} / \mathrm{R}$ & MS & $\mathrm{ZB}$ & 0.01 & 0.33 & 0.04 & 0.55 & 3.4 & 1.9 & 4 & 4 \\
\hline Muraenidae sp. & $\mathrm{R}$ & & & & 0.33 & & 1.98 & & 1.9 & & 4 \\
\hline Engraulidae & & & & & & & & & & & \\
\hline Anchoa lyolepis (Evermann and Marsh, 1900) & $\mathrm{D}$ & MS & $\mathrm{ZP}$ & 0.02 & & & & 3.4 & & 4 & \\
\hline Anchoa marinii Hildebrand, 1943 & $\mathrm{D}$ & MS & $\mathrm{ZP}$ & 0.04 & & 0.01 & & 3.4 & & 4 & \\
\hline Anchoa sp. & $\mathrm{R}$ & & & 0.06 & & 0.01 & & 3.4 & & 4 & \\
\hline Anchoa spinifer (Valenciennes, 1848) & $\mathrm{D}$ & MM & PV & 0.21 & & 0.04 & & 17.2 & & 4 & \\
\hline Anchoa tricolor (Spix and Agassiz, 1829) & $\mathrm{D} / \mathrm{R}$ & MM & $\mathrm{ZB}$ & 0.12 & & 0.03 & & 10.3 & & 4 & \\
\hline Anchovia clupeoides (Swainson, 1839) & $\mathrm{D}$ & MM & $\mathrm{ZP}$ & 0.91 & & 1.06 & & 3.4 & & 4 & \\
\hline Cetengraulis edentulus (Cuvier, 1829) & $\mathrm{D} / \mathrm{R}$ & MM & $\mathrm{ZP}$ & 4.63 & & 6.55 & & 41.4 & & 2 & \\
\hline Engraulis anchoita Hubbs and Marini, 1935 & $\mathrm{R}$ & MS & $\mathrm{ZP}$ & 0.25 & & 0.1 & & 3.4 & & 4 & \\
\hline Lycengraulis grossidens (Spix and Agassiz, 1829) & $\mathrm{D} / \mathrm{R}$ & $\mathrm{ES}$ & PV & 0.2 & & 0.05 & & 13.8 & & 4 & \\
\hline Clupeidae & & & & & & & & & & & \\
\hline Harengula clupeola (Cuvier, 1829) & $\mathrm{D} / \mathrm{R}$ & MS & $\mathrm{ZP}$ & 0.24 & & 0.44 & & 6.9 & & 4 & \\
\hline Opisthonema oglinum (Lesueur, 1818)* & $\mathrm{D} / \mathrm{R}$ & MS & $\mathrm{ZP}$ & 0.21 & 1.84 & 0.10 & 0.22 & 17.2 & 15.4 & 4 & 2 \\
\hline Rhinosardinia bahiensis (Steindachner, 1879) & $\mathrm{D} / \mathrm{R}$ & ES & $\mathrm{ZP}$ & 0.07 & & 0.02 & & 17.2 & & 4 & \\
\hline Sardinella brasiliensis (Steindachner, 1879) & $\mathrm{D} / \mathrm{R}$ & MS & $\mathrm{ZP}$ & 0.06 & & 0.05 & & 6.9 & & 4 & \\
\hline Chaetodontidae & & & & & & & & & & & \\
\hline Chaetodon ocellatus Bloch, 1787 & $\mathrm{D}$ & MS & $\mathrm{ZB}$ & 0.01 & & & & 3.4 & & 4 & \\
\hline Ariidae & & & & & & & & & & & \\
\hline Ariidae sp. & $\mathrm{D}$ & & & & 0.22 & & 0.27 & & 1.9 & & 4 \\
\hline Aspistor luniscutis (Valenciennes, 1840) & $\mathrm{D} / \mathrm{R}$ & MS & OV & & 5.31 & & 2.15 & & 15.4 & & 2 \\
\hline Aspistor quadriscutis (Valenciennes, 1840) & $\mathrm{D} / \mathrm{R}$ & MS & $\mathrm{ZB}$ & & 0.87 & & 0.4 & & 9.6 & & 4 \\
\hline Aspistor sp. & $\mathrm{R}$ & & & & 0.33 & & 0.13 & & 1.9 & & 4 \\
\hline Bagre marinus (Mitchill, 1815) & $\mathrm{D} / \mathrm{R}$ & $\mathrm{MM}$ & $\mathrm{ZB}$ & & 1.52 & & 0.92 & & 9.6 & & 2 \\
\hline Cathorops agassizii (Eigenmann and Eigenmann, 1888) & $\mathrm{R}$ & ES & $\mathrm{ZB}$ & 0.01 & & 0.04 & & 3.4 & & 4 & \\
\hline Cathorops spixii (Agassiz, 1829) & $\mathrm{R}$ & ES & $\mathrm{ZB}$ & & 0.43 & & 0.11 & & 3.8 & & 4 \\
\hline Sciades herzbergii (Bloch, 1794) & $\mathrm{D} / \mathrm{R}$ & ES & $\mathrm{ZB}$ & 0.07 & & 1.11 & & 10.3 & & 4 & \\
\hline Sciades proops (Valenciennes, 1840) & $\mathrm{D} / \mathrm{R}$ & ES & $\mathrm{ZB}$ & & 1.84 & & 2.29 & & 7.7 & & 2 \\
\hline Synodontidae & & & & & & & & & & & \\
\hline Synodus foetens (Linnaeus, 1766) & $\mathrm{D} / \mathrm{R}$ & MS & PV & 0.02 & & 0.02 & & 6.9 & & 4 & \\
\hline Batrachoididae & & & & & & & & & & & \\
\hline Batrachoides surinamensis (Bloch and Schneider, 1801) & $\mathrm{D} / \mathrm{R}$ & MS & $\mathrm{ZB}$ & 0.04 & & 0.21 & & 13.8 & & 4 & \\
\hline Thalassophryne nattereri Steindachner, 1876 & $\mathrm{D} / \mathrm{R}$ & MS & $\mathrm{ZB}$ & 0.08 & & 0.17 & & 20.7 & & 4 & \\
\hline Mugilidae & & & & & & & & & & & \\
\hline Mugil curema Valenciennes, $1836 *$ & $\mathrm{D} / \mathrm{R}$ & MM & DV & 10.4 & 0.65 & 41.8 & 0.4 & 17.2 & 9.6 & 2 & 4 \\
\hline Atherinopsidae & & & & & & & & & & & \\
\hline Atherinella brasiliensis (Quoy and Gaimard, 1825) & $\mathrm{D} / \mathrm{R}$ & ES & OV & 0.01 & & $* *$ & & 6.9 & & 4 & \\
\hline Belonidae & & & & & & & & & & & \\
\hline Tylosurus acus acus (Lacepède, 1803) & $\mathrm{D}$ & MS & PV & 0.02 & & 0.03 & & 10.3 & & 4 & \\
\hline Hemiramphidae & & & & & & & & & & & \\
\hline Hemiramphus brasiliensis (Linnaeus, 1758) & $\mathrm{R}$ & MS & HV & 0.10 & & 0.06 & & 13.8 & & 4 & \\
\hline Hyporhamphus unifasciatus (Ranzani, 1841) & $\mathrm{D} / \mathrm{R}$ & MM & $\mathrm{OV}$ & 0.10 & & 0.07 & & 20.7 & & 4 & \\
\hline Syngnathidae & & & & & & & & & & & \\
\hline Syngnathus sp. & $\mathrm{D}$ & & & 0.01 & & $* *$ & & 3.4 & & 4 & \\
\hline Triglidae & & & & & & & & & & & \\
\hline Prionotus punctatus (Bloch, 1793) & $\mathrm{D}$ & MS & $\mathrm{ZB}$ & 0.01 & & $* *$ & & 3.4 & & 4 & \\
\hline Centropomidae & & & & & & & & & & & \\
\hline Centropomus parallelus Poey, 1860 & $\mathrm{D} / \mathrm{R}$ & MM & PV & 0.46 & & 1.25 & & 20.7 & & 4 & \\
\hline Centropomus pectinatus Poey, 1860 & $\mathrm{D} / \mathrm{R}$ & MM & PV & 0.03 & & 0.09 & & 6.9 & & 4 & \\
\hline Centropomus sp. & $\mathrm{D}$ & & & & 0.11 & & 0.5 & & 1.9 & & 4 \\
\hline Centropomus undecimalis (Bloch, 1792)* & $\mathrm{D} / \mathrm{R}$ & MM & PV & 0.26 & 0.76 & 2.41 & 2.26 & 17.2 & 11.5 & 4 & 4 \\
\hline Serranidae & & & & & & & & & & & \\
\hline Epinephelus adscensionis (Osbeck, 1765) * & $\mathrm{D} / \mathrm{R}$ & MS & ZB & 0.01 & 0.11 & $* *$ & 0.01 & 3.4 & 1.9 & 4 & 4 \\
\hline Epinephelus marginatus (Lowe, 1834) & $\mathrm{R}$ & MS & OP & 0.01 & & 0.18 & & 3.4 & & 4 & \\
\hline Mycteroperca bonaci (Poey, 1860) * & $\mathrm{D}$ & MS & PV & 0.01 & 0.11 & $* *$ & 0.02 & 3.4 & 1.9 & 4 & 4 \\
\hline Carangidae & & & & & & & & & & & \\
\hline Carangoides bartholomaei (Cuvier, 1833) & $\mathrm{D} / \mathrm{R}$ & MS & PV & & 3.9 & & 1.51 & & 19.2 & & 2 \\
\hline Caranx crysos (Mitchill, 1815) * & $\mathrm{D}$ & MS & PV & 0.01 & 0.11 & $* *$ & 0.03 & 3.4 & 1.9 & 4 & 4 \\
\hline Caranx hippos (Linnaeus, 1766)* & $\mathrm{D} / \mathrm{R}$ & MS & PV & 0.4 & 6.39 & 0.24 & 40.5 & 17.2 & 32.7 & 4 & 2 \\
\hline Caranx latus Agassiz, 1831* & $\mathrm{D} / \mathrm{R}$ & MS & $\mathrm{ZB}$ & 0.21 & 0.98 & 0.33 & 3.63 & 17.2 & 9.6 & 4 & 4 \\
\hline Caranx ruber (Bloch, 1793) & $\mathrm{D}$ & MM & $\mathrm{ZB}$ & & 3.25 & & 0.54 & & 1.9 & & 2 \\
\hline
\end{tabular}


Table 1 (Cont.). - Composition of the ichthyofauna captured in the Itapissuma/Itamaracá Complex. D, dry; R, rainy; EUFG, estuarine use functional group; ES, estuarine species; MM, marine migrants; MS, marine stragglers; FMFG, feeding mode functional group; HV, herbivore; DV, detritivore; OV, omnivore; PV, piscivore; ZB, zoobenthivore; ZP, zooplanktivore; E, estuary; C, coast; N, abundance; B, biomass; FO, occurrence frequency; E, estuary; C, coast; IR, relative importance: 1 , abundant and frequent; 2 , abundant and infrequent; 4 , less abundant and infrequent; $(*)$ Species present in all the studied environments. Sea $=$ Season. $* *$ biomass $(\%)<0.01$

\begin{tabular}{|c|c|c|c|c|c|c|c|c|c|c|c|}
\hline \multirow{2}{*}{ Species } & \multirow{2}{*}{ Sea } & \multirow{2}{*}{\multicolumn{2}{|c|}{ EUFG FMFG }} & \multicolumn{2}{|c|}{$\mathrm{N}(\%)$} & \multicolumn{2}{|c|}{$\mathrm{B}(\%)$} & \multicolumn{2}{|c|}{$\mathrm{FO}(\%)$} & & \\
\hline & & & & $\mathrm{E}$ & $\mathrm{C}$ & $\mathrm{E}$ & $\mathrm{C}$ & $\mathrm{E}$ & $\mathrm{C}$ & $\mathrm{E}$ & $\mathrm{C}$ \\
\hline Chloroscombrus chrysurus (Linnaeus, 1766)* & $\mathrm{D} / \mathrm{R}$ & MS & $\mathrm{ZB}$ & 0.15 & 0.54 & 0.01 & 0.07 & 13.8 & 9.6 & 4 & 4 \\
\hline Oligoplites palometa (Cuvier, 1832)* & $\mathrm{D} / \mathrm{R}$ & MM & PV & 0.01 & 1.3 & $* *$ & 1.14 & 3.4 & 17.3 & 4 & 4 \\
\hline Oligoplites saliens (Bloch, 1793) & $\mathrm{D}$ & MM & PV & 0.01 & & 0.01 & & 3.4 & & 4 & \\
\hline Oligoplites saurus (Bloch and Schneider, 1801)* & $\mathrm{D} / \mathrm{R}$ & MM & PV & 0.02 & 0.87 & 0.01 & 0.25 & 10.3 & 11.5 & 4 & 4 \\
\hline Selene brownii (Cuvier, 1816) & $\mathrm{D} / \mathrm{R}$ & MS & $\mathrm{ZB}$ & & 19.5 & & 6.18 & & 48.1 & & 2 \\
\hline Selene spixii (Castelnau, 1855) & $\mathrm{R}$ & MS & $\mathrm{ZB}$ & & 0.76 & & 0.41 & & 3.8 & & 4 \\
\hline Selene vômer (Linnaeus, 1758) & $\mathrm{D} / \mathrm{R}$ & MS & PV & & 8.99 & & 5.36 & & 57.7 & & 1 \\
\hline Trachinotus carolinus (Linnaeus, 1766) & $\mathrm{D} / \mathrm{R}$ & MM & $\mathrm{ZB}$ & & 0.98 & & 3.03 & & 11.5 & & 4 \\
\hline Trachinotus falcatus (Linnaeus, 1758) & $\mathrm{D} / \mathrm{R}$ & MS & $\mathrm{ZB}$ & & 0.98 & & 3.6 & & 13.5 & & 4 \\
\hline Trachinotus goodei Jordan and Evermann, 1896 & $\mathrm{D} / \mathrm{R}$ & MS & $\mathrm{ZB}$ & & 0.76 & & 0.53 & & 7.7 & & 4 \\
\hline Lutjanidae & & & & & & & & & & & \\
\hline Lutjanus alexandrei Moura and Lindeman, 2007 & $\mathrm{D} / \mathrm{R}$ & MS & $\mathrm{ZB}$ & 0.28 & & 0.85 & & 17.2 & & 4 & \\
\hline Lutjanus analis (Cuvier, 1828)* & $\mathrm{D} / \mathrm{R}$ & MS & $\mathrm{ZB}$ & 0.41 & 1.08 & 0.13 & 0.45 & 41.4 & 13.5 & 4 & 4 \\
\hline Lutjanus jocu (Bloch and Schneider, 1801) * & $\mathrm{D} / \mathrm{R}$ & MS & $\mathrm{ZB}$ & 0.24 & 0.22 & 0.19 & 0.05 & 31 & 1.9 & 4 & 4 \\
\hline Lutjanus synagris (Linnaeus, 1758) & $\mathrm{D} / \mathrm{R}$ & MS & $\mathrm{ZB}$ & 0.33 & & 0.03 & & 17.2 & & 4 & \\
\hline Gerreidae & & & & & & & & & & & \\
\hline Diapterus auratus Ranzani, $1842 *$ & $\mathrm{D} / \mathrm{R}$ & MM & $\mathrm{ZB}$ & 1.44 & 2.17 & 4.08 & 0.79 & 20.7 & 21.2 & 2 & 2 \\
\hline Diapterus rhombeus (Cuvier, 1829) * & $\mathrm{D} / \mathrm{R}$ & MM & $\mathrm{ZP}$ & 1.11 & 0.43 & 0.55 & 0.28 & 41.4 & 3.8 & 2 & 4 \\
\hline Diapterus sp. & $\mathrm{R}$ & & & 0.06 & & 0.01 & & 6.9 & & 4 & \\
\hline Eucinostomus argenteus Baird and Girard, $1855 *$ & $\mathrm{D} / \mathrm{R}$ & MM & ZB & 4.69 & 0.33 & 5.75 & 0.07 & 75.9 & 5.8 & 1 & 4 \\
\hline Eucinostomus gula (Quoy and Gaimard, 1824) & $\mathrm{D} / \mathrm{R}$ & MM & $\mathrm{ZB}$ & 2.84 & & 1.99 & & 55.2 & & 1 & \\
\hline Eucinostomus havana (Nichols, 1912) & $\mathrm{D} / \mathrm{R}$ & MM & $\mathrm{ZB}$ & 0.18 & & 0.25 & & 17.2 & & 4 & \\
\hline Eucinostomus melanopterus (Bleeker, 1863) & $\mathrm{R}$ & $\mathrm{MM}$ & $\mathrm{ZB}$ & 0.07 & & 0.12 & & 3.4 & & 4 & \\
\hline Eucinostomus sp. & $\mathrm{D} / \mathrm{R}$ & & & 0.52 & & 0.05 & & 20.7 & & 4 & \\
\hline Eugerres brasilianus (Cuvier, 1830) & $\mathrm{D} / \mathrm{R}$ & MM & $\mathrm{OV}$ & 0.03 & & 0.01 & & 6.9 & & 4 & \\
\hline Haemulidae & & & & & & & & & & & \\
\hline Anisotremus moricandi (Ranzani, 1842) & $\mathrm{D} / \mathrm{R}$ & MS & $\mathrm{OV}$ & & 0.43 & & 0.06 & & 7.7 & & 4 \\
\hline Anisotremus virginicus (Linnaeus, 1758) & $\mathrm{R}$ & MS & $\mathrm{OV}$ & & 0.43 & & 0.08 & & 1.9 & & 4 \\
\hline Conodon nobilis (Linnaeus, 1758) & $\mathrm{D}$ & MM & $\mathrm{ZB}$ & & 0.22 & & 0.02 & & 1.9 & & 4 \\
\hline Genyatremus luteus (Bloch, 1790) & $\mathrm{R}$ & MS & $\mathrm{OP}$ & 0.02 & & 0.11 & & 3.4 & & 4 & \\
\hline Haemulon aurolineatum Cuvier, 1830 & $\mathrm{D}$ & MS & $\mathrm{ZB}$ & & 0.22 & & 0.06 & & 1.9 & & 4 \\
\hline Haemulon parra (Desmarest, 1823) & $\mathrm{D} / \mathrm{R}$ & MS & $\mathrm{ZB}$ & & 1.3 & & 0.53 & & 9.6 & & 4 \\
\hline Haemulon plumierii (Lacepède, 1801) & $\mathrm{D}$ & MS & $\mathrm{ZB}$ & & 6.18 & & 0.89 & & 11.5 & & 2 \\
\hline Haemulon steindachneri (Jordan and Gilbert, 1882) & $\mathrm{D} / \mathrm{R}$ & MS & $\mathrm{ZB}$ & & 0.43 & & 0.19 & & 5.8 & & 4 \\
\hline Pomadasys corvinaeformis (Steindachner, 1868) & $\mathrm{D} / \mathrm{R}$ & MS & $\mathrm{ZB}$ & & 2.93 & & 0.45 & & 11.5 & & 2 \\
\hline Pomadasys crocro (Cuvier, 1830) & $\mathrm{D} / \mathrm{R}$ & MS & $\mathrm{ZB}$ & 0.01 & & 0.07 & & 6.9 & & 4 & \\
\hline Sparidae & & & & & & & & & & & \\
\hline Archosargus probatocephalus (Walbaum, 1792) & $\mathrm{D}$ & MS & $\mathrm{OV}$ & 0.03 & & $* *$ & & 6.9 & & 4 & \\
\hline Archosargus rhomboidalis (Linnaeus, 1758) * & $\mathrm{D} / \mathrm{R}$ & MS & $\mathrm{ZB}$ & 0.81 & 0.54 & 0.19 & 0.32 & 27.6 & 7.7 & 2 & 4 \\
\hline Polynemidae & & & & & & & & & & & \\
\hline Polydactylus virginicus (Linnaeus, 1758) * & $\mathrm{D} / \mathrm{R}$ & MM & $\mathrm{ZB}$ & 0.02 & 3.14 & 0.05 & 1.01 & 6.9 & 3.8 & 4 & 2 \\
\hline Sciaenidae & & & & & & & & & & & \\
\hline Bairdiella ronchus (Cuvier, 1830) & $\mathrm{D} / \mathrm{R}$ & MM & $\mathrm{ZB}$ & 0.18 & & 0.62 & & 13.8 & & 4 & \\
\hline Cynoscion sp. & $\mathrm{D}$ & & & 0.03 & & $* *$ & & 3.4 & & 4 & \\
\hline Cynoscion virescens (Cuvier, 1830) & $\mathrm{D}$ & MM & $\mathrm{ZB}$ & 0.06 & & 0.01 & & 10.3 & & 4 & \\
\hline Isopisthus parvipinnis (Cuvier, 1830) & $\mathrm{R}$ & MM & PV & & 0.43 & & 0.39 & & 3.8 & & 4 \\
\hline Larimus breviceps Cuvier, 1830 & $\mathrm{R}$ & MM & $\mathrm{ZB}$ & & 0.33 & & 0.06 & & 1.9 & & 4 \\
\hline Menticirrhus americanus (Linnaeus, 1758) & $\mathrm{D} / \mathrm{R}$ & MM & $\mathrm{ZB}$ & & 0.43 & & 0.23 & & 5.8 & & 4 \\
\hline Ophioscion sp. & $\mathrm{D}$ & & & 0.01 & & 0.03 & & 3.4 & & 4 & \\
\hline Paralonchurus brasiliensis (Steindachner, 1875) & $\mathrm{D}$ & MM & $\mathrm{ZB}$ & & 0.33 & & 0.04 & & 1.9 & & 4 \\
\hline Stellifer stellifer (Bloch, 1790) & $\mathrm{D}$ & ES & $\mathrm{ZB}$ & 0.02 & & 0.03 & & 3.4 & & 4 & \\
\hline Mullidae & & & & & & & & & & & \\
\hline Pseudupeneus maculatus (Bloch, 1793) & $\mathrm{D}$ & MS & $\mathrm{ZB}$ & & 0.11 & & 0.02 & & 1.9 & & 4 \\
\hline Labridae & & & & & & & & & & & \\
\hline Halichoeres radiatus (Linnaeus, 1758) & $\mathrm{D}$ & MS & $\mathrm{ZB}$ & & 0.11 & & 0.02 & & 1.9 & & 4 \\
\hline Scaridae & & & & & & & & & & & \\
\hline Sparisoma radians (Valenciennes, 1840) & $\mathrm{R}$ & MS & $\mathrm{HV}$ & 0.65 & & 0.14 & & 1.9 & & 4 & \\
\hline Sparisoma axillare (Steindachner, 1878) * & $\mathrm{D} / \mathrm{R}$ & MS & $\mathrm{HV}$ & 0.23 & 0.65 & 0.04 & 0.09 & 10.3 & 7.7 & 4 & 4 \\
\hline Sparisoma cf amplum & $\mathrm{R}$ & MS & $\mathrm{HV}$ & & 0.33 & & 0.11 & & 3.8 & & 4 \\
\hline Ephippidae & & & & & & & & & & & \\
\hline Chaetodipterus faber (Broussonet, 1782) * & $\mathrm{D} / \mathrm{R}$ & MM & $\mathrm{OV}$ & 0.1 & 1.52 & 1.26 & 1.38 & 6.9 & 17.3 & 4 & 2 \\
\hline Pomacanthidae & & & & & & & & & & & \\
\hline Pomacanthus paru (Bloch, 1787) & $\mathrm{R}$ & MS & $\mathrm{ZP}$ & & 0.11 & & 0.01 & & 1.9 & & 4 \\
\hline Eleotridae & & & & & & & & & & & \\
\hline Guavina guavina (Valenciennes, 1837) & $\mathrm{D}$ & ES & $\mathrm{ZB}$ & 0.01 & & $* *$ & & 3.4 & & 4 & \\
\hline Gobiidae & & & & & & & & & & & \\
\hline Ctenogobius boleosoma (Jordan and Gilbert, 1882) & $\mathrm{D}$ & ES & DV & 0.13 & & 0.01 & & 3.4 & & 4 & \\
\hline Ctenogobius shufeldti (Jordan and Eigenmann, 1887) & $\mathrm{D} / \mathrm{R}$ & ES & $\mathrm{OV}$ & 0.17 & & 0.03 & & 20.7 & & 4 & \\
\hline Ctenogobius smaragdus (Valenciennes, 1837) & $\mathrm{D} / \mathrm{R}$ & ES & DV & 0.48 & & 0.08 & & 44.8 & & 4 & \\
\hline Ctenogobius stigmaticus (Poey, 1860) & $\mathrm{D} / \mathrm{R}$ & $\mathrm{ES}$ & DV & 3.83 & & 0.35 & & 48.3 & & 2 & \\
\hline Evorthodus lyricus (Girard, 1858) & $\mathrm{D}$ & MS & DV & 0.01 & & $* *$ & & 3.4 & & 4 & \\
\hline Gobionellus oceanicus (Pallas, 1770) & $\mathrm{D} / \mathrm{R}$ & $\mathrm{ES}$ & DV & 2.44 & & 4.01 & & 58.6 & & 1 & \\
\hline
\end{tabular}


$138 \cdot V$. Ferreira et al.

Table 1 (Cont.). - Composition of the ichthyofauna captured in the Itapissuma/Itamaracá Complex. D, dry; R, rainy; EUFG, estuarine use functional group; ES, estuarine species; MM, marine migrants; MS, marine stragglers; FMFG, feeding mode functional group; HV, herbivore; $\mathrm{DV}$, detritivore; OV, omnivore; PV, piscivore; ZB, zoobenthivore; ZP, zooplanktivore; E, estuary; C, coast; N, abundance; B, biomass; FO, occurrence frequency; E, estuary; C, coast; IR, relative importance: 1 , abundant and frequent; 2 , abundant and infrequent; 4 , less abundant and infrequent; (*) Species present in all the studied environments. Sea $=$ Season. $* *$ biomass $(\%)<0.01$.

\begin{tabular}{|c|c|c|c|c|c|c|c|c|c|c|c|}
\hline \multirow[b]{2}{*}{ Species } & \multirow[b]{2}{*}{ Sea } & \multirow{2}{*}{\multicolumn{2}{|c|}{ EUFG FMFG }} & \multicolumn{2}{|c|}{$\mathrm{N}(\%)$} & \multicolumn{2}{|c|}{$\mathrm{B}(\%)$} & \multicolumn{2}{|c|}{$\mathrm{FO}(\%)$} & \multicolumn{2}{|c|}{ IR } \\
\hline & & & & $\mathrm{E}$ & $\mathrm{C}$ & $\mathrm{E}$ & $\mathrm{C}$ & $\mathrm{E}$ & $\mathrm{C}$ & $\mathrm{E}$ & $\mathrm{C}$ \\
\hline Gobionellus stomatus Starks, 1913 & $\mathrm{D} / \mathrm{R}$ & ES & DV & 53.5 & & 18.5 & & 58.6 & & 1 & \\
\hline Microgobius meeki Evermann and Marsh, 1899 & $\mathrm{D}$ & MS & $\mathrm{ZB}$ & 0.11 & & & & 6.9 & & 4 & \\
\hline Trichiuridae & & & & & & & & & & & \\
\hline Trichiurus lepturus Linnaeus, 1758 & $\mathrm{D} / \mathrm{R}$ & MS & PV & & 7.8 & & 7.43 & & 44.2 & & 2 \\
\hline Acanthuridae & & & & & & & & & & & \\
\hline Acanthurus bahianus Castelnau, 1855 & $\mathrm{D} / \mathrm{R}$ & MS & $\mathrm{HV}$ & & 0.43 & & 0.07 & & 5.8 & & 4 \\
\hline Acanthurus chirurgus (Bloch, 1787) * & $\mathrm{D} / \mathrm{R}$ & MS & $\mathrm{HV}$ & 0.01 & 0.33 & & 0.03 & 6.9 & 3.8 & 4 & 4 \\
\hline Acanthurus coeruleus Bloch and Schneider, 1801 & $\mathrm{D}$ & MS & $\mathrm{HV}$ & & 0.11 & & 0.01 & & 1.9 & & 4 \\
\hline Sphyraenidae & & & & & & & & & & & \\
\hline Sphyraena barracuda (Edwards, 1771) & $\mathrm{D} / \mathrm{R}$ & MM & PV & 0.05 & & 0.38 & & 6.9 & & 4 & \\
\hline Sphyraena guachancho Cuvier, $1829 *$ & $\mathrm{D}$ & MS & PV & 0.02 & 0.22 & 0.19 & 0.18 & 6.9 & 1.9 & 4 & 4 \\
\hline Sphyraena viridensis Cuvier, 1829 & $\mathrm{D}$ & MS & PV & & 0.11 & & 0.12 & & 1.9 & & 4 \\
\hline Scombridae & & & & & & & & & & & \\
\hline $\begin{array}{l}\text { Scomberomorus brasiliensis Collette, Russo and Zavala- } \\
\text { Camin, } 1978\end{array}$ & $\mathrm{D}$ & MS & PV & & 0.22 & & 0.13 & & 1.9 & & 4 \\
\hline Paralichthyidae & & & & & & & & & & & \\
\hline Citharichthys sp. & $\mathrm{D} / \mathrm{R}$ & & & 0.11 & & 0.02 & & 10.3 & & 4 & \\
\hline Citharichthys spilopterus Günther, 1862 & $\mathrm{D} / \mathrm{R}$ & MM & $\mathrm{ZB}$ & 0.79 & & 0.26 & & 48.3 & & 4 & \\
\hline Etropus crossotus Jordan and Gilbert, 1882 & $\mathrm{R}$ & MM & $\mathrm{ZB}$ & 0.5 & & 0.06 & & 6.9 & & 4 & \\
\hline Paralichthys brasiliensis (Ranzani, 1842)* & $\mathrm{D} / \mathrm{R}$ & MM & $\mathrm{ZB}$ & 0.01 & 0.11 & 0.02 & 0.01 & 6.9 & 1.9 & 4 & 4 \\
\hline Syacium micrurum Ranzani, 1842 & $\mathrm{D}$ & MM & $\mathrm{ZB}$ & & 0.11 & & 0.01 & & 1.9 & & 4 \\
\hline Syacium papillosum (Linnaeus, 1758) & $\mathrm{D}$ & MS & $\mathrm{ZB}$ & & 0.11 & & 0.01 & & 1.9 & & 4 \\
\hline Bothidae & & & & & & & & & & & \\
\hline Bothus ocellatus (Agassiz, 1831) & $\mathrm{R}$ & MM & $\mathrm{ZB}$ & & 0.11 & & ** & & 1.9 & & 4 \\
\hline Achiridae & & & & & & & & & & & \\
\hline Achirus declivis Chabanaud, 1940 & $\mathrm{D}$ & ES & $\mathrm{ZB}$ & 0.03 & & $* *$ & & 10.3 & & 4 & \\
\hline Achirus lineatus (Linnaeus, 1758) & $\mathrm{D} / \mathrm{R}$ & ES & $\mathrm{ZB}$ & 1.48 & & 0.08 & & 48.3 & & 2 & \\
\hline Achirus sp. & $\mathrm{D} / \mathrm{R}$ & & & 0.68 & & 0.03 & & 13.8 & & 4 & \\
\hline Trinectes paulistanus (Miranda Ribeiro, 1915) & $\mathrm{D}$ & MM & ZB & 0.21 & & 0.01 & & 3.4 & & 4 & \\
\hline Cynoglossidae & & & & & & & & & & & \\
\hline Symphurus tessellatus (Quoy and Gaimard, 1824) & $\mathrm{D} / \mathrm{R}$ & MM & ZB & 0.04 & & 0.04 & & 17.2 & & 4 & \\
\hline Ostraciidae & & & & & & & & & & & \\
\hline $\begin{array}{l}\text { Lactophrys trigonus (Linnaeus, 1758) } \\
\text { (1) }\end{array}$ & $\mathrm{D}$ & MS & ZB & & 0.11 & & 0.28 & & 1.9 & & 4 \\
\hline Tetraodontidae & & & & & & & & & & & \\
\hline Colomesus psittacus (Bloch and Schneider, 1801) & $\mathrm{D} / \mathrm{R}$ & MS & ZB & 0.03 & & 1.43 & & 6.9 & & 4 & \\
\hline Sphoeroides greeleyi Gilbert, 1900 & $\mathrm{D} / \mathrm{R}$ & ES & $\mathrm{ZB}$ & 0.2 & & 0.05 & & 27.6 & & 2 & \\
\hline Sphoeroides testudineus (Linnaeus, 1758) & $\mathrm{D} / \mathrm{R}$ & ES & ZB & 2.13 & & 2.01 & & 79.3 & & 1 & \\
\hline Diodontidae & & & & & & & & & & & \\
\hline Chilomycterus spinosus spinosus (Linnaeus, 1758) & $\mathrm{R}$ & MS & ZB & & 0.11 & & 0.05 & & 1.9 & & 4 \\
\hline
\end{tabular}

effectively sampled: 88 species $(88 \%$ of the estimated richness) were observed in the estuary and 75 species ( $85 \%$ of the estimated richness) on the coast. A total of 25 species $(18 \%)$ were common to both the estuary and the coast, 65 species $(47 \%)$ were exclusive to the estuary and 50 species $(35 \%)$ occurred only on the coast (Table 1).

In the estuary, Engraulidae ( 9 species), Gerreidae (9 species) and Gobiidae ( 8 species) were dominant in richness (S). The Gobiidae family had the highest abundance $(\% \mathrm{~N})$ in the dry (7694 individuals, 62\%) and rainy (3776 individuals, 58\%) seasons. In terms of biomass, Mugilidae were dominant during the dry season (114.34 kg, 51.74\%) and Gobiidae during the rainy season $(22.20 \mathrm{~kg}$, $28 \%$ ). The gobiid Gobionelus stomatus Starks, 1913 showed the highest abundance in both seasons (dry season 6582 individuals, 53\%; rainy season 3532 individuals, 54\%), while for biomass, Mugil curema Valenciennes, 1836 (114.39 kg, 52\%) and Cetengraulis edentulus (Spix and Agassiz, 1829) $(18 \mathrm{~kg}, 22.42 \%)$ were dominant during the dry and the rainy seasons, respectively (Table 1 ).
On the coast, Carangidae were dominant in richness (14 species); in abundance, with 288 individuals $(49 \%)$ and 166 individuals $(50 \%)$ during the dry and rainy seasons, respectively; and in biomass, with $115 \mathrm{~kg}$ $(57 \%)$ and $203 \mathrm{~kg}(74 \%)$ in the dry and rainy seasons, respectively. In terms of species, Selene brownii (Cuvier, 1816) was dominant with the highest abundance d,uring the dry season (138 individuals; 23\%) and Selene vomer (Linnaeus, 1758) during the rainy season (45 individuals, 14\%), while Trichiurus lepturus Linnaeus, 1758 (26 kg, 13\%) and Caranx hippos (Linnaeus, 1766) (151.59 $\mathrm{kg}, 55 \%$ ) dominated in terms of biomass during the dry and the rainy seasons, respectively (Table 1).

Less abundant and infrequent species were dominant in the estuary (85\%) and on the coast $(77 \%)$ (Table 1). Eucinostomus argenteus Baird and Girard, 1855, Eucinostomus gula (Quoy and Gaimard, 1824), Ctenogobius smaragdus (Valenciennes, 1837), Ctenogobius stigmaticus (Poey, 1860), Gobionellus oceanicus (Pallas, 1770), G. stomatus and Sphoeroides testudineus (Linnaeus, 1758) were considered abundant and frequent in the estuary, and $S$. vomer in the coastal area. 


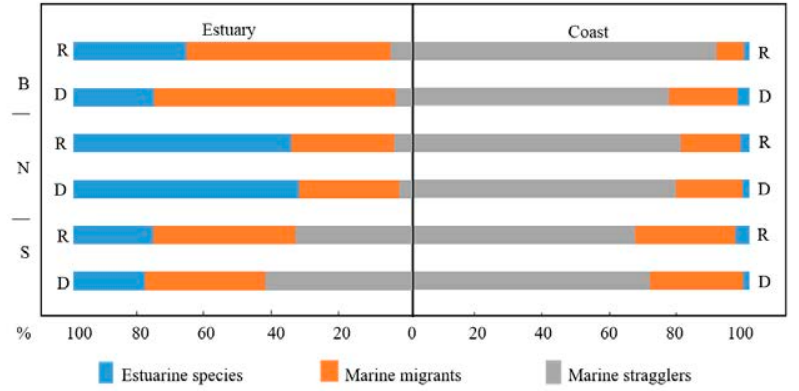

Fig. 2. - Percentage participation (\%) of richness (S), abundance $(\mathrm{N})$ and biomass (B) of estuarine use guilds by season (D, dry; R, rainy) and location in the Itapissuma/Itamaracá Complex, northeastern Brazil.

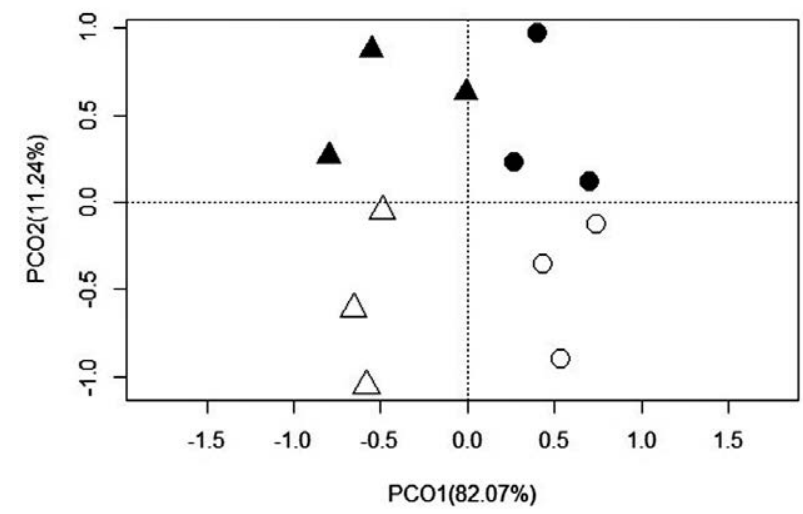

Fig. 3. - Principal coordinates ordination analysis of the richness of estuarine use guilds in the estuary (circle) and coast (triangle) during the dry (empty) and rainy (full) seasons in the Itapissuma/Itamaracá

$$
\text { Complex. }
$$

\section{Estuarine use structure}

Richness, abundance and biomass of the estuarine use guilds did not vary by season, but differences were observed between the estuary and the coast. In the estuary, marine stragglers and marine migrants dominated in richness during the dry season (33 species, 43\%) and the rainy (23 species, $41 \%$ ). Estuarine species showed the highest abundance in the dry season (8150 individuals, 66\%) and the rainy season (4099 individuals, 64\%), but in terms of biomass, marine migrants dominated throughout the year (Fig. 2). On the coast, marine stragglers were dominant in richness (38 species, 70\%; 31 species, 65\%), in abundance (458 individuals, 78\%; 259 individuals, $79 \%)$ and in biomass $(147 \mathrm{~kg}, 76 \% ; 238 \mathrm{~kg}$, $90 \%$ ) in the dry and rainy seasons, respectively (Fig. 2).

The PCO analysis based on the estuarine use guilds revealed that the main effect along the first axis (82.07 $\%)$ was spatial as it discriminated the samples from the coast and the estuary. Estuarine samples were very

Table 2. - PERMANOVA test results for the effects of environment and season on the richness of estuarine use guilds in the Itapissuma/ Itamaracá Complex, northeastern Brazil.

\begin{tabular}{lrllrl}
\hline & d.f & SS & MS & Pseudo-F & p \\
\hline Environment & 1 & 0.153 & 0.153 & 32.348 & $\mathbf{0 . 0 0 1}$ \\
Season & 1 & 0.017 & 0.017 & 3.592 & 0.069 \\
Environment vs. Season & 1 & 0.000 & 0.000 & 0.063 & 0.892 \\
Residuals & 8 & 0.037 & 0.004 & & \\
Total & 11 & 0.208 & & & \\
\hline
\end{tabular}

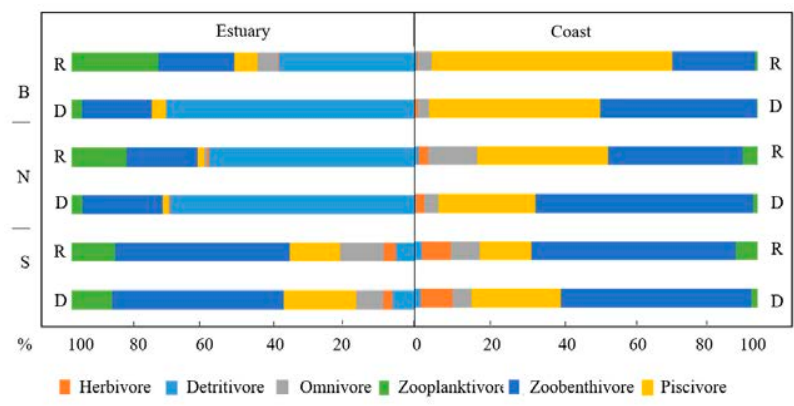

Fig. 4. - Percentage participation (\%) of richness (S), abundance (N) and biomass (B) of trophics guilds by season (D, dry; R, rainy) and location in the Itapissuma/Itamaracá Complex, northeastern Brazil.

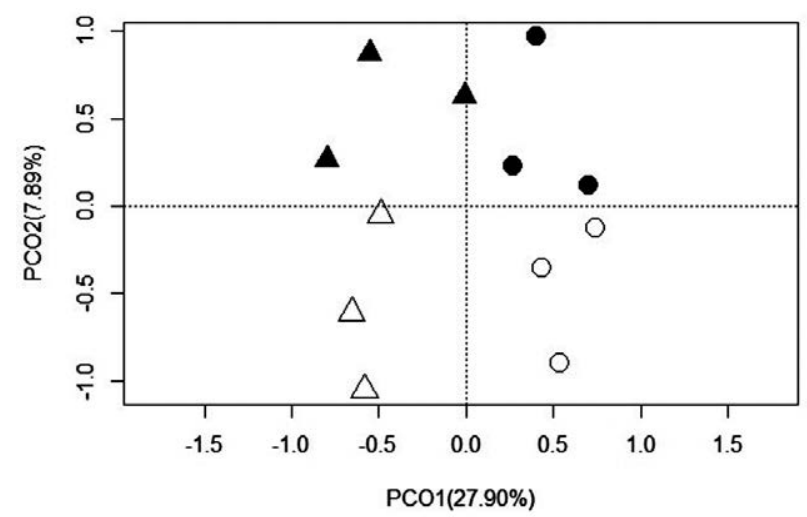

Fig. 5. - Principal coordinates ordination analysis of the richness of trophic guilds in the estuary (circle) and coast (triangle) during the dry (empty) and rainy (full) seasons in the Itapissuma/Itamaracá

$$
\text { Complex. }
$$

similar between seasons, whereas coastal samples showed a more heterogeneous pattern (Fig. 3). The patterns were tested through PERMANOVA and confirmed the location (estuary and coast) effect $(\mathrm{p}<0.05)$. No seasonal effect was observed (Table 2, $\mathrm{p}=0.01$ ).

\section{Trophic structure}

Zoobenthivores were the richest trophic guild in the estuary: 38 species $(41 \%)$ and 28 species $(30 \%)$ in the dry and rainy seasons, respectively. The detritivores showed the highest abundance (15452 individuals, $62 \%$; 10176 individuals, 53) and biomass (203 kg, 70; $82 \mathrm{~kg}, 47 \%$ ) in the dry and rainy seasons, respectively (Fig. 4). On the coast, zoobenthivores also dominated in richness (30 species, 55.5\%; 34 species, 60.1\%) and abundance (372 individuals, 63.3\%; 229 individuals, $50 \%$ ), and piscivores had the greatest biomass (96 kg, $49 \% ; 188 \mathrm{~kg}, 66.9 \%$ ) in the dry and rainy seasons, respectively (Fig. 4).

Table 3. - PERMANOVA test results on the richness of trophic guilds, testing for the effects of factors environment and season in the Itapissuma/Itamaracá Complex, northeastern Brazil.

\begin{tabular}{lrllrc}
\hline & d.f & SS & MS & Pseudo-F & p \\
\hline Environment & 1 & 0.077 & 0.077 & 6.919 & $\mathbf{0 . 0 0 4}$ \\
Season & 1 & 0.016 & 0.016 & 1.450 & 0.273 \\
Environment vs. Season & 1 & 0.001 & 0.001 & 0.168 & 0.925 \\
Residuals & 8 & 0.089 & 0.011 & & \\
Total & 11 & 0.184 & & & \\
\hline
\end{tabular}


The PCO based on trophic guilds discriminated samples from the estuary and from the coast along axis $1(69.64 \%)$. In the estuary, differences were observed between the dry and rainy seasons (Fig. 5).

According to the PERMANOVA, the environments (estuary and coast) significantly influenced the abundance of the trophic guilds in the IIC (Table 3, p=0.004), confirming the groups formed by PCO (Fig. 5).

\section{DISCUSSION}

Overall, species composition of the IIC was similar to that of the fish fauna typically found in other tropical estuaries (Paiva et al. 2008, Mourão et al. 2014). The observed species richness was close to the estimated richness, indicating that sampling was satisfactory, thanks to the concomitant implementation of active and passive fishing gear. Sampling is known to affect catch composition, especially its diversity (Magurran and McGill 2011), but the use of different gears provides the best estimate of structure (Kwak and Peterson 2007, Mourão et al. 2014) and diversity of fish assemblages (Mérigot et al. 2016). Different gears use different capture processes, mainly based on fish behaviour (Huse et al. 1999). In this study, the use of multiple gears was necessary, considering the differential characteristics of each environment sampled and the fact that fish species explore different habitats of a given environment differently. By exploring multiple gears in different habitats, we improved the estimation of biodiversity, thus providing as wide a variety of guilds as possible.

PCO analysis and PERMANOVA showed spatial differences in the estuarine use functional group between the estuarine and coastal areas, but temporal variations were not evidenced. Spatial segregation processes were observed in other tropical estuaries (Mourão et al. 2014, Loureiro et al. 2016) and may be related to differences in the life cycle and in species tolerance to diverse environmental stresses. Temporal changes in the composition of estuarine fish communities were not observed in the IIC, as reported in other tropical estuaries (Castillo-Rivera et al. 2002, Mendoza et al. 2009).

In the estuary, migrant species predominated in richness and biomass, and estuarine species in abundance. The high richness and biomass of marine species in the estuary can be attributed to the permanent connection between the estuarine area and the Atlantic Ocean throughout the year (Medeiros and Kjerfve 1993), allowing an uninterrupted connectivity with the marine ecosystem (Vasconcelos et al. 2015). Migratory species are of great importance in connected systems, such as estuaries and the adjacent marine area (Harrison and Whitfield 2008). In addition, the IIC is considered a system with high biodiversity and primary and secondary productivity (Vasconcelos Filho et al. 2010, Mérigot et al. 2016). The positive effect of primary productivity on species richness allows larger populations to persist, thereby reducing extinction risk and supporting a higher diversity of niche specialists (Tittensor et al. 2010). According to Vasconcelos
Filho and Oliveira (1999), marine species of the IIC are mostly juveniles, some of which are of commercial value. The high abundance of estuarine species within the estuary of the IIC was mainly due to gobiids. Mérigot et al. (2016) analysed the diversity of fish communities in estuarine complexes in Brazil and revealed differences between assemblages from Itapissuma, especially due to the relatively high abundance of some species of Gobiidae. The high abundance of gobiids in tropical estuaries may be partly due to their prolonged larval duration (Shen and Tzeng 2008), closely linked to the mainly muddy substrate and thus restricting their migrations to the sea (Vasconcelos Filho and Oliveira 1999).

In the coastal environment of the IIC, the marine stragglers predominated in richness, abundance and biomass in all periods. However, the percentages of resident (estuarine) and dependent (marine migrant) species were also high, thus confirming the dependence between the estuary and coast of the IIC. The connection between continental and marine environments is an essential characteristic, as marine species are important exporters of energy to the adjacent coastal areas (Vasconcelos Filho et al. 2009). Also, the coast of the IIC offers favourable conditions for the development of the marine fish fauna as protection and food resource (Medeiros et al. 2001).

In relation to the feeding guild approach, our findings emphasized that the substrate of the IIC is of extreme importance for the high productivity in the system (CPRH 2010), contributing to the high occurrence of species with feeding habits associated with the substrate (i.e. zoobenthivores and detritivores). The high availability of organic rich detritus in mangroves may increase the feeding opportunities for detritivores (Kuo et al. 1999), and can be considered the main trophic contribution factor for the estuarine fish fauna (Paiva et al. 2008). In north Brazil, Loureiro et al. (2016) observed that fish assemblage was strongly associated with substrates composed of organic matter. The high richness of zoobenthivores in the estuarine area of the IIC can be attributed to the great abundance of available benthic fauna (Silva 2013). Benthos is one of the structuring elements of the food web and plays an important role in the system dynamics (Herman et al. 1999), transferring energy to fishes in estuarine environments (Buchheister and Latour 2015). Detrivores dominated in abundance and biomass mainly due to large supply of organic matter and detritus in the IIC (Vasconcelos Filho et al. 2009, 2010), which support estuarine trophic webs (Hoffman et al. 2008). The estuarine organic material of the IIC originates from various rivers (Eskinazi-Lessa et al. 1999). The river discharge, sediment resuspension, mangrove litter, waste input, terrestrial runoff and atmospheric input are sources of nutrients in the IIC estuary (Medeiros 1991). The highest proportion of detritus usually occurs in environments with great amounts of organic matter. Detritus is consumed, constituting a link between organic production and animal nutrition, and increasing the efficiency of the energy transfer between the trophic levels (Qasim and Sankaranarayanan 1972). 
The large supply of zoobenthic fauna (Silva 2013) and the sandy substrate along the coast (Almeida and Manso 2011) favour the high species richness and abundance of zoobenthivores in the IIC coastal area. Benthophagous fish are highly associated with sandy substrates (Loureiro et al. 2016). The dominance of piscivores in biomass is mainly due to large carangids, which benefit from a high supply of food in the coastal area. Carangids are visual, active predators that spend a great part of their time on the reef searching for prey (Cervigón 1972): they feed on fish and also consume benthic prey to complement their diets (MorenoSánchez et al. 2016).

Estuaries are dynamic ecosystems subject to notable variability of environmental conditions, and their fish assemblages show within-estuary seasonal and spatial variations, so taking into account this variability should further clarify trait patterns and drivers of estuarine fish (Henriques et al. 2017). The IIC is an important ecosystem for several species that inhabit or visit the area, mainly associated with the substrate. However, coastal areas are exposed to multiple anthropogenic pressures (Blaber and Barletta 2016) that can alter the structure and function of the fish community (Baptista et al. 2015). The anthropogenic stresses and climate changes may facilitate or inhibite the processing of detritus and consequently cause dramatic shifts in species composition, which are often long-lasting and difficult to reverse (Ooi and Chong 2011). The increase in human impacts could significantly affect the topology and functioning of the food web by altering stabilizing elements of the network and decreasing the diversity of trophic flows that ensures the resilience of the trophic structure (Lobry et al. 2008). From the point of view of ecosystem management, it is necessary to identify and understand the biotic and abiotic effects on the distribution of fish fauna as a precursor for the management and monitoring of coastal environments (Pichler et al. 2017).

\section{ACKNOWLEDGEMENTS}

The first author thanks Coordenação de Aperfeiçoamento de Pessoal de Nível Superior (CAPES) for the graduate scholarship and $\mathrm{CNPq}$ (Conselho Nacional de Desenvolvimento Científico) for the sandwich PhD scholarship. The last author also thanks $\mathrm{CNPq}$ for a research grant. The authors wish to thank the colleagues who assisted in the field and laboratory work. This work was financially supported by CAPESCOFECUB (Process 88881.142689/2017-01) and CNPq and the Instituto Nacional de Ciência e Tecnologia - Ambientes Marinhos Tropicais (CNPq Process 565054).

\section{REFERENCES}

Able K.W., Fahay M.P., Witting D.A., et al. 2005. Fish settlement in the ocean vs. estuary: Comparison of pelagic larval and settled juvenile composition and abundance. Estuar. Coast. Shelf Sci. 66: 280-290.

https://doi.org/10.1016/j.ecss.2005.09.003

Almeida T.L.M., Manso V.A.V. 2011. Sedimentologia da plata- forma interna adjacente a Ilha de Itamaracá - Pe. Est. Geol. 21: 135-152.

Anderson M.J. 2001. A new method for non-parametric multivariate analysis of variance. Austral Ecol. 26: 32-46. https://doi.org/10.1111/j.1442-9993.2001.01070.pp.x

Angel A., Ojeda F.P. 2001. Structure and trophic organization of subtidal fish assemblages on the northern Chilean coast: the effect of habitat complexity. Mar. Ecol. Prog. Ser. 217: 81-91. https://doi.org/10.3354/meps217081

Baptista J., Martinho F., Nyitrai D., et al. 2015. Long-term functional changes in an estuarine fish assemblage. Mar. Pollut. Bull. 97: 125-134. https://doi.org/10.1016/j.marpolbul.2015.06.025

Blaber S.J.M., Barletta M. 2016. A review of estuarine fish research in South America: what has been achieved and what is the future for sustainability and conservation? J. Fish Biol. 89: 537-568. https://doi.org/10.1111/jfb.12875

Buchheister A., Latour R.J. 2015. Diets and trophic-guild structure of a diverse fish assemblage in Chesapeake Bay, U.S.A. J. Fish Biol. 86: 967-992. https://doi.org/10.1111/jfb.12621

Castillo-Rivera M., Zavala-Hurtado J.A., Zárate R. 2002. Exploration of spatial and temporal patterns of fish diversity and composition in a tropical estuarine system of Mexico. Rev. Fish Biol. Fish. 12: 167-177. https://doi.org/10.1023/A:1025051027676

Cervigón F. 1972. Los peces. In: Ginés H., Margalef R. (eds), Ecología Marina. Dossat S. A, Caracas, pp. 308-355.

Colwell R.K. 2013. EstimateS: Statistical estimation of species richness and shared species from samples. Version 9. User's Guide and application at http://purl.oclc.org/estimates

Colwell R.K., Mao C.X., Chang J. 2004. Interpolating, extrapolating, and comparing incidence-based species accumulation curves. Ecology 85: 2717-2727.

CPRH. Agência Estadual de Meio Ambiente. 2010. Diagnóstico Sócio ambiental da Área de Protecão Ambiental de Santa Cruz. Companhia Pernambucana de Meio Ambiente, Recife, 388 pp.

Elliott M., Quintino V. 2007. The estuarine quality paradox, environmental homeostasis and the difficulty of detecting anthropogenic stress in naturally stressed areas. Mar. Pollut. Bull. 54: 640-645. https://doi.org/10.1016/j.marpolbul.2007.02.003

Elliott M., Whitfield A.K., Potter I.C., et al. 2007. The guild approach to categorizing estuarine fish assemblages: A global review. Fish Fish. 8: 241-268. https://doi.org/10.1111/j.1467-2679.2007.00253.x

Eskinazi-Leça E., Barros H.M., Macedo S.J. 1999. Estuarine management in northeastern Brazil: the estuarine complex of Itamaracá. Trans. Ecol. Environ. 27: 247-256.

Feyrer F., Cloern J.E., Brown L.R., et al. 2015. Estuarine fish communities respond to climate variability over both river and ocean basins. Glob. Chang. Biol. 21: 3608-3619. https://doi.org/10.1111/gcb. 12969

Froese R., Pauly D. 2007. FishBase. Version 06/2018. http:// www.fishbase.org

Garcia A.M., Vieira J.P. 2001. O aumento da diversidade de peixes no estuário da Lagoa dos Patos durante o episódio El Niño 1997-1998. Atlântica 23: 133-152.

Garrison L.P., Link J. 2000. Fishing effects on spatial distribution and trophic guild structure of the fish community in the Georges Bank region. ICES J. Mar. Sci. 57: 723-730. https://doi.org/10.1006/jmsc.2000.0713

Gray J.S., Elliott M. 2009. Ecology of Marine Sediments: From Science to Management. Oxford Univ. Press, NY, $256 \mathrm{pp}$.

Harrison T.D., Whitfield A.K. 2008. Geographical and typological changes in fish guilds of South African estuaries. J. Fish Biol. 73: $2542-2570$. https://doi.org/10.1111/j.1095-8649.2008.02108.x

Henriques S., Guilhaumon F., Villéger S. 2017. Biogeographical region and environmental conditions drive functional traits of estuarine fish assemblages worldwide. Fish Fish. 18: 752-771. https://doi.org/10.1111/faf.12203

Herman P.M.J., Middelburg J.J., van de Koppel J., et al. 1999. Ecology of Estuarine Macrobenthos. Adv. Ecol. Res. 29: 195-240. https://doi.org/10.1016/S0065-2504(08)60194-4

Hoffman J.C., Bronk D.A., Olney J.E. 2008. Organic matter sources supporting lower food web production in the tidal freshwater portion of the York River estuary, Virginia. Estuaries and Coasts 31: 898-911. https://doi.org/10.1007/s12237-008-9073-4 
Huse I., Gundersen A.C., Nedreaas K.H. 1999. Relative selectivity of Greenland halibut (Reinhardtius hippoglossoides, Walbaum) by trawls, longlines and gillnets. Fish. Res. 44: 75-93. https:// doi.org/10.1016/S0165-7836(99)00041-7

Kempf M. 1970. Nota preliminar sobre os fundos costeiros da região de Itamaracá (Norte do Estado de Pernambuco, Brasil). Trab. Oceanog. Univ. Fed. Pernambuco 11: 95-111.

Kuo S., Lin H., Shao K. 1999. Fish assemblages in the mangrove creeks of northern and southern Taiwan. Estuaries and Coasts 22: $1004-1015$. https://doi.org/10.2307/1353079

Kwak T.J., Peterson J.T. 2007. Community indices, parameters, and comparisons. In: Guy C.S., Brown M.L. (eds), Analysis and interpretation of freshwater fisheries data. American Fisheries Society, Maryland, pp. 677-763.

Lobry J., David V., Pasquaud S., et al. 2008. Diversity and stability of an estuarine trophic network. Mar. Ecol. Prog. Ser. 358: 13-25. https://doi.org/10.3354/meps07294

Loureiro S.N., Reis-Filho J.A., Giarrizzo T. 2016. Evidence for habitat-driven segregation of an estuarine fish assemblage. J. Fish Biol. 55: 804-820. https://doi.org/10.1111/jfb.13017

Macêdo S.J., Flores Montes M.J., Lins I.C. 2000. Características abióticas da área. In: Barros H.M., Eskinazi-Leça E., Macêdo S.J., et al. (eds), Gerenciamento participativo de estuários e manguezais. UFPE, Recife, pp. 7-25.

Magurran A.E., McGill B.J. 2011. Biological Diversity - Frontiers in Measurement and Assessment. Oxford Univ. Press, New York, $345 \mathrm{pp}$.

Medeiros C. 1991. Circulation and mixing processes in the Itamaracá estuarine system, Brazil. Ph.D. thesis, Univ. South Carolina, $131 \mathrm{pp}$.

Medeiros C., Kjerfve B. 1993. Hidrology of a tropical estuarine system: Itamaracá, Brazil. Estuar. Coast. Shelf Sci. 36: 495-515.

Medeiros C., Kjerfve B., Araújo Filho M., et al. 2001. The Itamaracá Estuarine Ecosystem, Brazil. In: Seelinger U., Kjerfve B. (eds), Ecological Studies: Coastal Marine Ecosystems of Latin America. Springer-Verlag, New York, pp. 71-81.

Mendoza E., Castillo-Rivera M., Zárate-Hernández R., et al. 2009. Seasonal variations in the diversity, abundance, and composition of species in an estuarine fish community in the Tropical Eastern Pacific, Mexico. Ichthyol. Res. 56: 330-339. https://doi.org/10.1007/s10228-009-0102-5

Mérigot B., Frédou F.L., Viana A.P., et al. 2016. Fish assemblages in tropical estuaries of northeastern Brazil: A multi-component diversity approach. Ocean Coast. Manag. 143: 175-183. https://doi.org/10.1016/j.ocecoaman.2016.08.004

Moreno-Sánchez X.G., Palacios-Salgado D.S., Abitia-Cárdenas L.A., et al. 2016. Importance of benthos in the trophic structure of the ichthyofauna of Los Frailes reef, Gulf of California, Mexico. In: Riosmena-Rodriguez R. (ed), Marine benthos: biology, ecosystem functions, and environmental impact. Nova Science Publishers, New York, pp. 1-37.

Mourão K.R.M., Ferreira V., Lucena-Frédou F. 2014. Composition of functional ecological guilds of the fish fauna of the internal sector of the amazon estuary, pará, Brazil. An. Acad. Bras. Cienc. 86: 1783-1800. https://doi.org/10.1590/0001-3765201420130503

Nelson J.S., Grande T.C., Wilson M.V.H. 2016. Fishes of the world. Wiley \& Sons, New Jersey, 299 pp.

Ooi A.L., Chong V.C. 2011. Larval fish assemblages in a tropical mangrove estuary and adjacent coastal waters: offshore-inshore flux of marine and estuarine species. Cont. Shelf Res. 31: 1599-1610. https://doi.org/10.1016/j.csr.2011.06.016

Paiva A.C.G., Coelho P.A., Torres M.F.A. 2005. Influência dos fatores abióticos sobre a macrofauna de substratos inconsolidados da zona entre-marés no canal de Santa Cruz, Pernambuco, Brasil. Arq. Ciên. Mar. 38: 85-92.

Paiva A.C.G., Chaves P.D.T.D.C., Araújo M.E. 2008. Estrutura e organização trófica da ictiofauna de águas rasas em um estuário tropical. Rev. Bras. Zool. 25: 647-661. https://doi.org/10.1590/S0101-81752008000400010

Pichler H.A., Gary C.A., Broadhurst M.K., et al. 2017. Seasonal and environmental influences on recruitment patterns and habitat usage among resident and transient fishes in a World Heritage Site subtropical estuary. J. Fish Biol. 90: 396-416. https://doi.org/10.1111/jfb.13191

Qasim S.Z., Sankaranarayanan V.N. 1972. Organic detritus of a tropical estuary. Mar. Biol. 15: 193-199.

R Core Team. 2018. R: A language and environment for statistical computing. R Foundation for Statistical Computing, Vienna, Austria.

Ray G.C. 2005. Connectivities of estuarine fishes to the coastal realm. Estuar. Coast. Shelf Sci. 64: 18-32. https://doi.org/10.1016/j.ecss.2005.02.003

Root R.B. 1967. The niche exploitation pattern of the blue-gray gnatcatcher. Ecol. Monographs 37: 317-350.

Shen K., Tzeng W. 2008. Reproductive strategy and recruitment dynamics of amphidromous goby Sicyopterus japonicus as revealed by otolith microstructure. J. Fish Biol. 73: 2497-2512. https://doi.org/10.1111/j.1095-8649.2008.02102.x

Silva A.M.C. 2013. Composição da meiofauna na ilha de Itamaracá e sua relação com a descrição morfoscópica e morfométrica dos grãos, Pernambuco. Rev. Nord. Zool. 7: 34-47.

Smith E.P., van Belle G. 1984. Nonparametric Estimation of Species Richness. Biometrics 40: 119-129. https://doi.org/10.2307/2530750

Tittensor D., Mora C., Jetz W., et al. 2010. Global patterns and predictors of marine biodiversity across taxa. Nature 466: 1098-1101. https://doi.org/10.1038/nature09329

Vasconcelos R.P., Henriques S., França S., et al. 2015. Global patterns and predictors of fish species richness in estuaries. J. Anim. Ecol. 84: 1331-1341 https://doi.org/10.1111/1365-2656.12372

Vasconcelos Filho A.L., Oliveira A.M.E. 1999. Composição e ecologia da ictiofauna do Canal de Santa Cruz (Itamaracá-PE, Brasil). Trab. Ocean. UFPE 27: 101-113.

Vasconcelos Filho A.L., Neumann-Leitão S., Eskinazi-Leça E., et al. 2003. Trophic interactions between fish and other compartment communities in a tropical estuary in Brazil as indicator of environmental quality. Adv. Ecol. Sci. 18: 173-183.

Vasconcelos Filho A.L., Neumann-Leitão S., Eskinazi-Leça E., et al. 2009. Hábitos alimentares de consumidores primários da ictiofauna do sistema estuarino de Itamaracá (Pernambuco Brasil). Rev. Bras. Eng. Pesca 4: 21-31.

Vasconcelos Filho A.L., Neumann-Leitão S., Eskinazi-Leça E., et al. 2010. Hábitos alimentares de peixes consumidores secundários do Canal de Santa Cruz, Pernambuco, Brasil. Trop. Oceanogr. 38: 121-128. https://doi.org/10.5914/tropocean.v38i2.5166

WoRMS Editorial Board. 2019. World Register of Marine Species. Accesed on 10/02/2019, at http://www.marinespecies.org/

\section{SUPPLEMENTARY MATERIAL}

The following supplementary material is available through the online version of this article and at the following link: http://scimar.icm.csic.es/scimar/supplm/sm04855esm.pdf

Fig. S1. - Species accumulation curve of the estuary (A) and coast (B), computed by a random method without replacement. Mean species richness value \pm SD.

Table S1. - Data collection dates according to the environmental and type of fishing gear utilised in the Itapissuma/Itamaracá Complex, northeastern Brazil.

Table S2. - Literature utilised for classication of the ecologic guilds of the ichthyofauna captured in the Itapissuma/Itamaracá Complex, northeastern Brazil. EUFG-Estuarine Use Functional Groups; FMFG-Feeding Mode Functional Groups, basead Elliott et al. (2007). 
Scientia Marina 83(2)

June 2019, S1-S7, Barcelona (Spain)

ISSN-L: 0214-8358

\section{Composition of the fish fauna in a tropical estuary: the ecological guild approach}

Valdimere Ferreira, François Le Loc’h, Frédéric Ménard, Thierry Frédou, Flávia L. Frédou

Supplementary material 


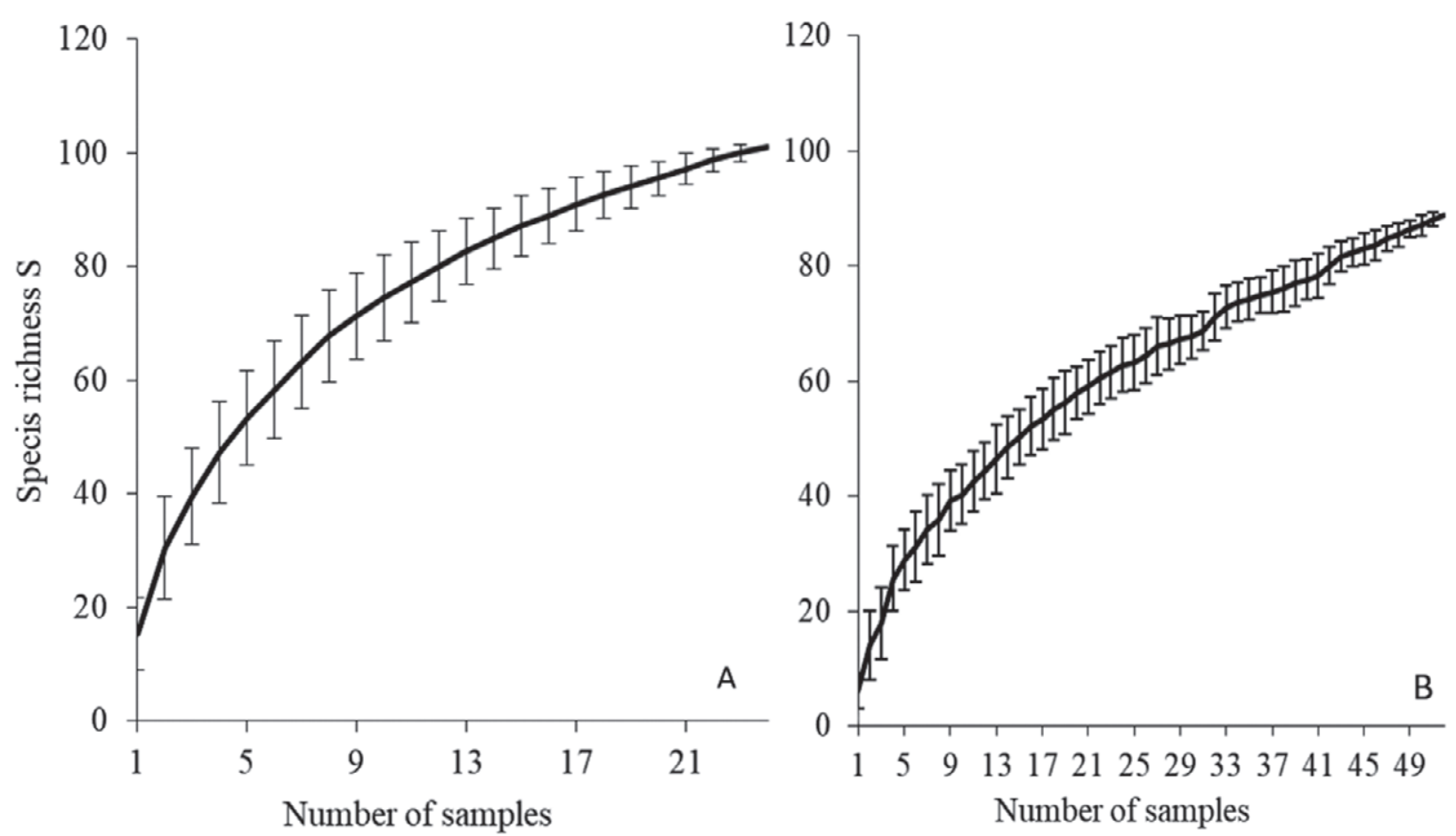

Fig. S1. - Species accumulation curve of the estuary (A) and coast (B), computed by a random method without replacement. Mean species richness value $\pm \mathrm{SD}$.

Table S1. - Data collection dates according to the environmental and type of fishing gear utilised in the Itapissuma/Itamaracá Complex, northeastern Brazil.

\begin{tabular}{|c|c|c|c|c|}
\hline Environmental & Season & Fishing gear & Date & Set \\
\hline \multirow[t]{12}{*}{ Estuary } & \multirow[t]{6}{*}{ Dry } & \multirow[t]{3}{*}{ Block net } & January-13 & 1 \\
\hline & & & November-13 & 1 \\
\hline & & & March-14 & 1 \\
\hline & & \multirow[t]{3}{*}{ Seine net } & January-13 & 3 \\
\hline & & & November-13 & 3 \\
\hline & & & March-14 & 3 \\
\hline & \multirow[t]{6}{*}{ Rainy } & \multirow[t]{3}{*}{ Block net } & May-13 & 1 \\
\hline & & & August-13 & 1 \\
\hline & & & May-14 & 1 \\
\hline & & \multirow[t]{3}{*}{ Seine net } & May-13 & 3 \\
\hline & & & August-13 & 3 \\
\hline & & & May-14 & 3 \\
\hline \multirow[t]{9}{*}{ Coast } & \multirow[t]{6}{*}{ Dry } & \multirow[t]{3}{*}{ Gill net } & February-13 & 3 \\
\hline & & & November-13 & 3 \\
\hline & & & March-14 & 3 \\
\hline & & \multirow[t]{3}{*}{ Tidal fixed trap } & February-13 & 6 \\
\hline & & & November-13 & 6 \\
\hline & & & February-14 & 6 \\
\hline & \multirow[t]{3}{*}{ Rainy } & \multirow[t]{3}{*}{ Gill net } & May-13 & 3 \\
\hline & & & August-13 & 3 \\
\hline & & & June-14 & 3 \\
\hline
\end{tabular}


Table S2. $-\neq$ Literature utilised for classication of the ecologic guilds of the ichthyofauna captured in the Itapissuma/Itamaracá Complex, northeastern Brazil. EUFG-Estuarine Use Functional Groups; FMFG-Feeding Mode Functional Groups, basead Elliott et al. (2007).

\begin{tabular}{|c|c|c|}
\hline \multirow{2}{*}{ Species } & \multicolumn{2}{|c|}{ Reference } \\
\hline & EUFG & FMFG \\
\hline Rhizoprionodon porosus & Lessa and Almeida 1997 & Lessa and Almeida 1997 \\
\hline Rhizoprionodon lalandii & Silva and Almeida 2001 & Bornatowski et al. 2014 \\
\hline Hypanus guttatus & Vasconcelos Filho and Oliveira 1999 & Gianeti 2011 \\
\hline Hypanus marianae & Shibuya and Rosa 2011 & Shibuya and Rosa 2011 \\
\hline Elops saurus & Vasconcelos Filho and Oliveira 1999 & Froese and Pauly 2019 \\
\hline Gymnothorax funebris & Vasconcelos Filho and Oliveira 1999 & Froese and Pauly 2019 \\
\hline Gymnothorax ocellatus & Froese and Pauly 2019 & Santos and Castro 2003 \\
\hline Anchoa lyolepis & Froese and Pauly 2019 & Froese and Pauly 2019 \\
\hline Anchoa marinii & Froese and Pauly 2019 & Froese and Pauly 2019 \\
\hline Anchoa spinifer & Vasconcelos Filho and Oliveira 1999 & Nizinski and Munroe 2002 \\
\hline Anchoa tricolor & Araújo et al. 2008 & Araújo et al. 2008 \\
\hline Anchovia clupeoides & Vasconcelos Filho and Oliveira 1999 & Paiva et al. 2008 \\
\hline Cetengraulis edentulus & Vasconcelos Filho and Oliveira 1999 & Paiva et al. 2008 \\
\hline Engraulis anchoita & Froese and Pauly 2019 & Vasconcellos et al. 1998 \\
\hline Lycengraulis grossidens & Mai and Vieira 2013 & Bortoluzzi et al. 2006 \\
\hline Harengula clupeola & Vasconcelos Filho and Oliveira 1999 & Paiva et al. 2008 \\
\hline Opisthonema oglinum & Vasconcelos Filho and Oliveira 1999 & Vasconcelos Filho 1979 \\
\hline Rhinosardinia bahiensis & Clark and Pessanha 2015 & Clark and Pessanha 2015 \\
\hline Sardinella brasiliensis & Castello 2007 & Castello 2007 \\
\hline Chaetodon ocellatus & Froese and Pauly 2019 & Froese and Pauly 2019 \\
\hline Aspistor luniscutis & Denadai et al. 2012 & Denadai et al. 2012 \\
\hline Aspistor quadriscutis & Denadai et al. 2012 & Denadai et al. 2012 \\
\hline Bagre marinus & Segura-Berttolini and Mendoza-Carranza 2013 & Mendonza-Carranza 2003 \\
\hline Cathorops agassizii & Dantas 2012 & Dantas 2012 \\
\hline Cathorops spixii & Vasconcelos Filho and Oliveira 1999 & Possato 2010 \\
\hline Sciades herzbergii & Vasconcelos Filho and Oliveira 1999 & Possato 2010 \\
\hline Sciades proops & Vasconcelos Filho and Oliveira 1999 & Guedes and Vasconcelos Filho 1980 \\
\hline Synodus foetens & Vasconcelos Filho and Oliveira 1999 & Cruz-Escalona et al. 2005 \\
\hline Batrachoides surinamensis & Froese and Pauly 2019 & Collette 2010 \\
\hline Thalassophryne nattereri & Vasconcelos Filho and Oliveira 1999 & Sampaio and Nottingham 2008 \\
\hline Guavina guavina & Vasconcelos Filho and Oliveira 1999 & Teixeira 1994 \\
\hline Ctenogobius boleosoma & Vasconcelos Filho and Oliveira 1999 & Vasconcelos Filho et al. 2009 \\
\hline Ctenogobius shufeldti & Wyanski and Targett 2000 & Contente et al. 2012 \\
\hline Ctenogobius smaragdus & Vasconcelos Filho and Oliveira 1999 & Lima 2015 \\
\hline Ctenogobius stigmaticus & Vasconcelos Filho and Oliveira 1999 & Lima 2015 \\
\hline Evorthodus lyricus & Vasconcelos Filho and Oliveira 1999 & STRI 2017 \\
\hline Gobionellus oceanicus & Vasconcelos Filho and Oliveira 1999 & Vasconcelos Filho et al. 2009 \\
\hline Gobionellus stomatus & Vasconcelos Filho and Oliveira 1999 & Lima 2015 \\
\hline Microgobius meeki & WoRMS Editorial Board 2019 & Froese and Pauly 2019 \\
\hline Mugil curema & Vasconcelos Filho and Oliveira 1999 & Medeiros 2013 \\
\hline Atherinella brasiliensis & Vasconcelos Filho and Oliveira 1999 & Paiva et al. 2008 \\
\hline Tylosurus acus acus & WoRMS Editorial Board 2019 & Froese and Pauly 2019 \\
\hline Hemiramphus brasiliensis & Vasconcelos Filho and Oliveira 1999 & Schwamborn 2004 \\
\hline Hyporhamphus unifasciatus & Vasconcelos Filho and Oliveira 1999 & Trigueiro 2013 \\
\hline Carangoides bartholomaei & Froese and Pauly 2019 & Paiva et al. 2008 \\
\hline Caranx crysos & Froese and Pauly 2019 & Sley et al. 2009 \\
\hline Caranx hippos & Vasconcelos Filho and Oliveira 1999 & Temóteo et al. 2015 \\
\hline Caranx latus & Vasconcelos Filho and Oliveira 1999 & Temóteo et al. 2015 \\
\hline Caranx ruber & Froese and Pauly 2019 & Froese and Pauly 2019 \\
\hline Chloroscombrus chrysurus & Vasconcelos Filho and Oliveira 1999 & Silva and Lopes 2002 \\
\hline Oligoplites palometa & Vasconcelos Filho and Oliveira 1999 & Vasconcelos Filho et al. 2010 \\
\hline Oligoplites saliens & Vasconcelos Filho and Oliveira 1999 & Winik et al. 2007 \\
\hline Oligoplites saurus & Vasconcelos Filho and Oliveira 1999 & Vasconcelos Filho et al. 2010 \\
\hline Selene brownii & WoRMS Editorial Board 2019 & Bomfim 2014 \\
\hline Selene spixii & WoRMS Editorial Board 2019 & Froese and Pauly 2019 \\
\hline Selene vômer & Vasconcelos Filho and Oliveira 1999 & Daros 2014 \\
\hline Trachinotus carolinus & Denadai et al. 2013 & Stefanoni 2008 \\
\hline Trachinotus falcatus & Vasconcelos Filho and Oliveira 1999 & Höflin et al. 1998 \\
\hline Trachinotus goodei & WoRMS Editorial Board 2019 & Stefanoni 2008 \\
\hline Sphyraena barracuda & Vasconcelos Filho and Oliveira 1999 & Akadje et al. 2013 \\
\hline Sphyraena guachancho & Bonecker et al. 2014 & Froese and Pauly 2019 \\
\hline Sphyraena viridensis & Barreiros et al. 2002 & Barreiros et al. 2002 \\
\hline Citharichthys spilopterus & Vasconcelos Filho and Oliveira 1999 & Vasconcelos Filho et al. 2010 \\
\hline Etropus crossotus & Oliveira and Favarro 2011 & Paiva et al. 2008 \\
\hline Paralichthys brasiliensis & Vasconcelos Filho and Oliveira 1999 & Froese and Pauly 2019 \\
\hline Syacium micrurum & Vasconcelos Filho and Oliveira 1999 & Lucato 1997 \\
\hline Syacium papillosum & Lucato 1997 & Lucato 1997 \\
\hline Lutjanus alexandrei & Fernandes et al. 2012 & Moraes 2012 \\
\hline Lutjanus analis & Vasconcelos Filho and Oliveira 1999 & Freitas et al. 2011 \\
\hline Lutjanus jocu & Vasconcelos Filho and Oliveira 1999 & Monteiro et al. 2009 \\
\hline Lutjanus synagris & Vasconcelos Filho and Oliveira 1999 & Froese and Pauly 2019 \\
\hline Diapterus auratus & Vasconcelos Filho and Oliveira 1999 & Temóteo 2015 \\
\hline Diapterus rhombeus & Vasconcelos Filho and Oliveira 1999 & Temóteo 2015 \\
\hline Eucinostomus argenteus & Vasconcelos Filho and Oliveira 1999 & Leão 2016 \\
\hline Eucinostomus gula & Vasconcelos Filho and Oliveira 1999 & Zahorcsak et al. 2000 \\
\hline
\end{tabular}


Table S2 (Cont.). - Literature utilised for classication of the ecologic guilds of the ichthyofauna captured in the Itapissuma/Itamaracá Complex, northeastern Brazil. EUFG-Estuarine Use Functional Groups; FMFG-Feeding Mode Functional Groups, basead Elliott et al. (2007).

\begin{tabular}{|c|c|c|}
\hline \multirow{2}{*}{ Species } & \multicolumn{2}{|c|}{ Reference } \\
\hline & EUFG & FMFG \\
\hline Eucinostomus havana & Vasconcelos Filho and Oliveira 1999 & Froese and Pauly 2019 \\
\hline Eucinostomus melanopterus & Chaves and Bouchereau 2000 & Araújo et al. 2016 \\
\hline Eugerres brasilianus & Vasconcelos Filho and Oliveira 1999 & Vasconcelos Filho et al. 2009 \\
\hline Anisotremus moricandi & Dias 2007 & Dias 2007 \\
\hline Anisotremus virginicus & Vasconcelos Filho and Oliveira 1999 & Dias 2007 \\
\hline Conodon nobilis & Vasconcelos Filho and Oliveira 1999 & Lira et al. $2013 \mathrm{a}$ \\
\hline Genyatremus luteus & Vasconcelos Filho and Oliveira 1999 & Almeida et al. 2005 \\
\hline Haemulon aurolineatum & Vasconcelos Filho and Oliveira 1999 & Dantas 2012 \\
\hline Haemulon parra & Vasconcelos Filho and Oliveira 1999 & Paiva et al. 2008 \\
\hline Haemulon plumierii & Shinozaki-Mendes et al. 2013 & Costa e Silva 2015 \\
\hline Haemulon steindachneri & Daros 2014 & Daros 2014 \\
\hline Pomadasys corvinaeformis & Vasconcelos Filho and Oliveira 1999 & Denadai et al. 2013 \\
\hline Pomadasys crocro & Froese and Pauly 2019 & Froese and Pauly 2019 \\
\hline Polydactylus virginicus & Vasconcelos Filho and Oliveira 1999 & Lopes and Oliveira-Silva 1998 \\
\hline Bairdiella ronchus & Vasconcelos Filho and Oliveira 1999 & Pina et al. 2015 \\
\hline Cynoscion virescens & Froese and Pauly 2019 & Froese and Pauly 2019 \\
\hline Isopisthus parvipinnis & Silva Junior et al. 2015 & Lira et al. $2013 \mathrm{~b}$ \\
\hline Larimus breviceps & Bessa et al. 2014 & Bessa et al. 2014 \\
\hline Menticirrhus americanus & Haluch et al. 2011 & Lira et al. 2013c \\
\hline Paralonchurus brasiliensis & Silva Junior et al. 2015 & Lira et al. 2013d \\
\hline Stellifer stellifer & Dantas 2012 & Pombo et al. 2013 \\
\hline Pseudupeneus maculatus & Vasconcelos Filho and Oliveira 1999 & Dantas 2012 \\
\hline Halichoeres radiatus & Froese and Pauly 2019 & Froese and Pauly 2019 \\
\hline Sparisoma radians & Vasconcelos Filho and Oliveira 1999 & Paiva et al. 2008 \\
\hline Sparisoma axillare & Feitosa and Ferreira 2014 & Feitosa and Ferreira 2014 \\
\hline Sparisoma aff. amplum & Francini-Filho et al. 2008 & Francini-Filho et al. 2008 \\
\hline Chaetodipterus faber & Froese and Pauly 2019 & Vasconcelos Filho et al. 2009 \\
\hline Pomacanthus paru & Vasconcelos Filho and Oliveira 1999 & Cerqueira and Haimovici 1990 \\
\hline Prionotus punctatus & Vasconcelos Filho and Oliveira 1999 & Longo et al. 2015 \\
\hline Centropomus parallelus & Vasconcelos Filho and Oliveira 1999 & Lira et al. 2016 \\
\hline Centropomus pectinatus & Jackson and Bockelmann-lobello 2006 & Lira et al. 2016 \\
\hline Centropomus undecimalis & Vasconcelos Filho and Oliveira 1999 & Lira et al. 2016 \\
\hline Epinephelus adscensionis & Nelson 2006 & Medeiros et al. 2017 \\
\hline Epinephelus marginatus & Andrade et al. 2003 & Machado et al. 2008 \\
\hline Mycteroperca bonaci & Daros 2014 & Daros 2014 \\
\hline Trichiurus lepturus & Vasconcelos Filho and Oliveira 1999 & Vasconcelos Filho et al. 2010 \\
\hline Scomberomorus brasiliensis & Vasconcelos Filho and Oliveira 1999 & Menezes 1970 \\
\hline Bothus ocellatus & Vasconcelos Filho and Oliveira 1999 & Hostim-Silva et al. 2005 \\
\hline Achirus declivis & Vasconcelos Filho and Oliveira 1999 & Couto and Farias 2001 \\
\hline Achirus lineatus & Vasconcelos Filho and Oliveira 1999 & Vasconcelos Filho et al. 2003 \\
\hline Trinectes paulistanus & Vasconcelos Filho and Oliveira 1999 & Contente et al. 2009 \\
\hline Symphurus tessellatus & Pina 2009 & Lima 2012 \\
\hline Acanthurus bahianus & Vasconcelos Filho and Oliveira 1999 & Pimentel 2012 \\
\hline Acanthurus chirurgus & Vasconcelos Filho and Oliveira 1999 & Longo et al. 2015 \\
\hline Acanthurus coeruleus & Longo et al. 2015 & Longo et al. 2015 \\
\hline Archosargus probatocephalus & Castillo-Rivera et al. 2007 & Castillo-Rivera et al. 2007 \\
\hline Archosargus rhomboidalis & Vasconcelos Filho and Oliveira 1999 & Yáñez-Arancibia et al. 1986 \\
\hline Lactophrys trigonus & Paiva et al. 2008 & Froese and Pauly 2019 \\
\hline Colomesus psittacus & Vasconcelos Filho and Oliveira 1999 & Araújo 2012 \\
\hline Sphoeroides greeleyi & Schultz 2002 & Lima 2014 \\
\hline Sphoeroides testudineus & Vasconcelos Filho and Oliveira 1999 & Vasconcellos et al. 1998 \\
\hline Chilomycterus spinosus & Vasconcelos Filho and Oliveira 1999 & Almeida-Silva et al. 2015 \\
\hline
\end{tabular}

Akadje C., Diaby M., Le Loc'h F., et al. 2013. Diet of the barracuda Sphyraena guachancho in Côte d'Ivoire (Equatorial Eastern Atlantic Ocean). Cybium 37: 285-293.

Almeida Z.S, Nunes J.L.S., Alves M.G.F.S. 2005. Dieta alimentar de Genyatremus luteus (Bloch, 1790) - (Teleostei, Perciformes: Haemulidae) na baía de São José, Maranhão, Brasil. Atlântica 27: 39-47.

Almeida-Silva P.H., Tubino R.A., Zambrano L.C., et al. 2015. Trophic ecology and food consumption of fishes in a hypersaline tropical lagoon. J. Fish Biol. 86: 1781-1795. https://doi.org/10.1111/jfb.12689

Andrade A.B., Machado L.F., Hostim-Silva M., et al. 2003. Reproductive biology of the dusky grouper Epinephelus marginatus (Lowe, 1834). Braz. Arch. Biol. Technol. 46: 373-381. https://doi.org/10.1590/S1516-89132003000300009

Araújo P.R.V. 2012. Variacão espaço-temporal e ecologia trófica de Sphoeroides testudineus (Linnaeus, 1758) e Colomesus psittacus (Bloch \& Schneider, 1801) (Actinopterygii, Tetraodontidae) no estuário do rio Mamanguape, Paraíba, Brasil. Graduation thesis, Univ. Est. Paraíba, 55 pp.

Araújo F.G., Silva M.A., Azevedo M.C. C., et al. 2008. Spawning season, recruitment and early life distribution of Anchoa tricolor (Spix and Agassiz, 1829) in a tropical bay in southeastern Brazil. Braz. J. Biol. 68: 823-829.

Araújo A.L.F., Dantas L.P., Pessanha A.L.M. 2016. Feeding ecology of three juvenile mojarras (Gerreidae) in a tropical estuary of northeastern Brazil. Neotrop. Ichthyol. 14: e150039. https://doi.org/10.1590/1982-0224-20150039

Barreiros J.P., Santos R.S., Barbosa A.E. 2002. Food habits, schooling and predatory behaviour of the yellowmouth barracuda Sphyraena viridensis, (Perciformes: Sphyraenidae) in the Azores. Cybium 26: 83-88.

Bessa E., Santos F.B., Pombo M., et al. 2014. Population ecology, life history and diet of the shorthead drum Larimus breviceps in a tropical 
bight in southeastern Brazil. J. Mar. Biol. Assoc. U.K. 94: 615-622. https://doi.org/10.1017/S0025315413001690

Bonecker A.C.T., Namiki C.A.P., Castro M.S.P., et al. 2014. Catálogo dos estágios iniciais de desenvolvimento dos peixes da bacia de Campos. Sociedade Brasileira de Zoologia, Curitiba, 296 pp.

Bomfim A.C. 2014. Bioecologia da ictiofauna marinha descartada pelo arrasto camaroeiro em praias da bacia potiguar, Brasil. Master thesis, Univ. Fed. Rio Grande do Norte, $127 \mathrm{pp}$.

Bornatowski H., Navia A.F., Braga R.R., et al. 2014. Ecological importance of sharks and rays in a structural foodweb analysis in Southern Brazil. ICES J. Mar. Sci. 71: 1586-1592. https://doi.org/10.1093/icesjms/fsu025

Bortoluzzi T., Aschenbrenner A.C., Silveira C.R., et al. 2006. Hábito alimentar da sardinha prata, Lycengraulis grossidens (Spix \& Agassiz, 1829), (Pisces, Engraulididae), Rio Uruguai médio, sudoeste do Rio Grande do Sul, Brasil. Biod. Pampeana 4: 11-23.

Castello J.P. 2007. Síntese sobre a distribuição, abundância, potencial pesqueiro e biologia da sardinha verdadeira (Sardinella brasiliensis). In: Haimovici M. (ed), A prospecção pesqueira e abundância de estoques marinhos no Brasil nas décadas de 1960 a 1990: Levantamento de dados e avaliação crítica. MMÃ/SMCQ, Brasília, p. 15.

Castillo-Rivera M., Zarate-Hernandez R., Salgado-Ugarte I. 2007. Juvenile and adult food habits of Archosargus probatocephalus (Teloostei : Sparidae) in a tropical estuary of Veracruz. Hidrobiologica 17: 119-126.

Cerqueira V.R., Haimovici M. 1990. Dinâmica populacional do gordinho, Peprilus paru (Pisces, Stromateidae), no litoral sul do Brasil. Rev. Bras. Biol. 50: 599-613.

Chaves P.T.C., Bouchereau J.L. 2000. Use of mangrove habitat for reproductive activity by the fish assemblage in the Guaratuba Bay, Brasil. Oceanol. Acta 23: 273-280

Clark F.J.K., Pessanha A.L.M. 2015. Diet and ontogenetic shift in habitat use by Rhinosardinia bahiensis in a tropical semi-arid estuary, North-Eastern Brazil. J. Mar. Biolog. Assoc. U.K. 95: 175-183. https://doi.org/10.1017/S0025315414000939

Collette B.B. 2010. Batrachoides surinamensis. IUCN Red List of Threatened Species Version 2018-1. https://doi.org/10.2305/IUCN.UK.2010-4.RLTS.T154930A4670747.en

Contente R.F., Stefanoni M.F., Spach H.L. 2009. Size-related changes in diet of the slipper sole Trinectes paulistanus (Actinopterygii, Achiridae) juveniles in a subtropical Brazilian estuary. Panam. J. Aquat. Sci. 4: 63-69.

Contente R.F., Stefanoni M.F., Spach H.L. 2012. Feeding ecology of the American freshwater goby Ctenogobius shufeldti (Gobiidae, Perciformes) in a sub-tropical estuary. J. Fish. Biol. 80: 2357-2373. https://doi.org/10.1111/j.1095-8649.2012.03300.x

Costa e Silva G.F. 2015. Ecologia alimentar e reprodução de Haemulon plumieri (Lacepéde, 1801) (Teleostei - Haemulidae) no Banco dos Abrolhos, Bahia - Brasil. Master thesis, Univ. Fed. Paraná, 52 pp.

Couto E.C.G., Farias M.C.V. 2001. Feeding habits of Sole Fish (Achirus declivis (Chabanaud, 1940) (Teleostei: Soleidae)) in the River Sergipe estuary (northeastern, Brazil). XII Coloq. Arg. Oceanogr., Mar Del Plata, Argentine.

Cruz-Escalona V.H., Peterson M.S., Campos-Dávila L., et al. 2005. Feeding habits and trophic morphology of inshore lizardfish (Synodus foetens) on the central continental shelf off Veracruz, Gulf of Mexico. J. Appl. Ichth. 21: 525-530. https://doi.org/10.1111/j.1439-0426.2005.00651.x

Daros F.A.L.M. 2014. Estudo da ictiofauna costeira no litoral do Paraná e adjacências através de censos visuais e microquímica de otólitos. Ph.D thesis, Univ. Fed. Paraná, 114 pp.

Dantas D.V. 2012. A utilização dos diferentes habitats do estuário do Rio Goiana pelas diferentes fases ontogenéticas das espécies Cathorops spixii, Cathorops agassizii, Stellifer brasiliensis e Stellifer stellifer (Actinopterygii, Teleostei). Estudo de caso: Caracterização das áreas utilizadas como berçário. Ph.D thesis, Univ. Fed. Pernambuco, 229 pp.

Denadai M.R., Bessa E., Santos F.B., et al. 2012. Life history of three catfish species (Siluriformes : Ariidae) from southeastern Brazil Life history of three catfish species (Siluriformes: Ariidae) from southeastern Brazil. Biota Neotrop. 12: 0-10.

Denadai M.R., Santos F.B., Bessa E., et al. 2013. Population biology and diet of the pompano Trachinotus carolinus (Perciformes: Carangidae) in Caraguatatuba Bay, Southeastern Brazil. J. Mar. Biol. Oceanogr. 2: 1-6. https://doi.org/10.4172/2324-8661.1000108

Elliott M., Whitfield A.K., Potter I.C., et al. 2007. The guild approach to categorizing estuarine fish assemblages: A global review. Fish Fish. 8: $241-268$ https://doi.org/10.1111/j.1467-2679.2007.00253 x

Dias T.L.P. 2007. What do we know about Anisotremus moricandi (Teleostei: Haemulidae), an endangered reef fish? Biota Neot. 7: $317-319$.

Francini-Filho R.B., Moura R.L., Ferreira C.M., et al. 2008. Live coral predation by parrotfishes (Perciformes: Scaridae) in the Abrolhos Bank, eastern Brazil, with comments on the classification of species into functional groups. Neotrop. Ichthyol. 6: 191-200.

Feitosa J.L., Ferreira B.P. 2014. Distribution and feeding patterns of juvenile parrotfish on algal-dominated coral reefs. Mar. Ecol. 36: 1-13. https://doi.org/10.1111/maec.12154

Fernandes C.A.F., Oliveira P.G.V., Travassos P.E.F., et al. 2012. Reproduction of the Brazilian snapper, Lutjanus alexandrei Moura \& Lindeman, 2007 (Perciformes: Lutjanidae), off the northern coast of Pernambuco, Brazil. Neotrop. Ichth. 10: 587-592. https://doi.org/10.1590/S1679-62252012005000022

Freitas M.O., Abilhoa V., Costa Silva G.H. 2011. Feeding ecology of Lutjanus analis (Teleostei: Lutjanidae) from Abrolhos Bank, Eastern Brazil. Neotrop. Ichth. 9: 411-418. https://doi.org/10.1590/S1679-62252011005000022

Froese R., Pauly D. 2019. FishBase. World Wide Web eletronic publication.

Gianeti M.D. 2011. Reprodução, alimentação, idade e crescimento de Dasyatis guttata (Bloch \& Schneider, 1801) (Elasmobranchii: Dasyatidae) na região de Caiçara do Norte - RN. Ph.D thesis, Univ. São Paulo, 131 pp.

Guedes D.S., Vasconcelos Filho A.L. 1980. Estudo ecológico da região de Itamaracá, Pernambuco, Brasil. Informações sobre alimentação dos bagres branco e amarelo (Pisces, Ariidae). Trab. Oceanogr. Univ. Fed. Pernambuco 15: 323-330. https://doi.org/10.5914/tropocean. 15 i1.2580

Haluch C.F., Abilhoa V., Freitas M.O., et al. 2011. Estrutura populacional e biologia reprodutiva de Menticirrhus americanus (Linnaeus, 1758) (Teleostei, Sciaenidae) na baía de Ubatuba-Enseada, Santa Catarina, Brasil. Biotemas 24: 47-59. https://doi.org/10.5007/2175-7925.2011v24n1p47

Höflin J.C., Ferreira L.I., Ribeiro-Neto F.B., et al. 1998. Alimentação de peixes da família Carangidae do complexo estuarino-lagunar de Cananéia, São Paulo, Brasil. Bioikos 12: 7-18.

Hostim-Silva M., Andrade A.B., Machado L.F., et al. 2005. Peixes de Costão Rochoso de Santa Catarina: I Arvoredo. Editora UNIVALI, Itajaí, $130 \mathrm{pp}$

Jackson T.L., Ockelmann-Lobello L. 2006. Centropomidae: Snooks. In: Richards W.J. (ed.), Early Stages of Atlantic Fishes - An Identification Guide for Western Central North Atlantic. CRC Taylor and Francis, Boca Raton, pp. 1197-1205.

Leão G.N. 2016. Aspectos da biologia de Eucinostomus argenteus Baird e Girard, 1855, Gerreidae, capturado no canal de Santa Cruz, Pernambuco. Master thesis, Univ. Fed. Rural. Pernambuco, 73 pp.

Lessa R.P., Almeida Z.S. 1997. Analysis of stomach contents of the smalltail shark, Carcharhinus porosus, from northern Brazil. Cybium 21: 123-133. 
Lima C.S.S. 2014. Partição trófica de dois Tetraodontidae simpatricos no estuário negativo do rio Tubarão, Macaú-RN, Brasil. Graduation thesis, Univ. Est. Paraíba, 56 pp.

Lima L.G. 2012. Ecologia trófica de Symphurus tessellatus (Quoy \& Gaimard, 1824) e Citharichthys macrops Dresel, 1885 (Actinopterygii, Pleuronectiformes) no sistema estuarino do rio Mamanguape, Paraíba - Brasil. Graduation thesis, Univ. Est. Paraíba, 43 pp.

Lima R.S. 2015. Ecologia alimentar de cinco gobídeos no estuário do rio Mamanguape, Paraíba. Graduation thesis, Univ. Est. Paraíba, 41pp.

Lira A.S., Frédou F.L., Viana A.P., et al. 2016. Feeding ecology of Centropomus undecimalis (Bloch, 1792) and Centropomus parallelus (Poey, 1860) in two tropical estuaries in Northeastern Brazil. Panam. J. Aquat. Sci. 12: 123-135.

Lira A.S., Viana A.P., Frédou F.L., et al. 2013a. Determinação do hábito alimentar do peixe roncador, Conodon nobilis (Linnaeus, 1758), na região de Barra de Sirinhaém-PE. IV Congr. Bras. Biol. Mar., Florianópolis, Brazil.

Lira A.S., Viana A.P., Fredou, F.L., et al. 2013b. Determinação do hábito alimentar de isophisthus parvipinis (Curvier, 1830) (Actinopterygii: Sciaenidae) na região de Barra de Sirinhaém PE. XVIII Congr. Bras. Eng. Pesca, Pernambuco, Brazil.

Lira A.S., Viana A.P., Nolé L.E., et al. 2013c. Hábito alimentar do Menticirrhus americanus (Linnaeus, 1758) (Actinopterygii: Sciaenidae) na região de Barra de Sirinhaém PE. XVIII Congr. Bras. Eng. Pesca, Pernambuco, Brazil.

Lira A.S., Viana A.P., Fay J., et al. 2013d. Determinação do hábito alimentar de Paralonchurus brasilienses (Steindachner, 1875) (Actinopterygii: Sciaenidae) na região de Barra de Sirinhaém PE. XVIII Congr. Bras. Eng. Pesca, Pernambuco, Brazil.

Longo G.O., Morais R.A., Martins C.D.L., et al. 2015. Between-habitat variation of benthic cover, reef fish assemblage and feeding pressure on the benthos at the only atoll in South Atlantic: Rocas atoll, NE Brazil. PLoS ONE 10: 1-29. https://doi.org/10.1371/journal.pone.0127176

Lopes P.R.D., Oliveira-Silva J.T. 1998. Nota sobre a alimentação de Conodon nobilis (Linnaeus) e Polydactylus virginicus (Linnaeus) (Actinopterygii: Haemulidae e Polynemidae) na praia de Jaguaribe, (Ilha de Itamaracá), Estado de Pernambuco. Rev. Bioikos 12: 53-59.

Lucato S.H.B. 1997. Trofodinâmica dos peixes Pleuronectiformes do Canal de São Sebastião, São Paulo, Brasil. Master thesis, Univ. São Paulo, $110 \mathrm{pp}$.

Machado L.F., Daros F.A.L.M., Bertoncini A.N., et al. 2008. Feeding strategy and trophic ontogeny in Epinephelus marginatus (Serranidae) from Southern Brazil. Cybium 32: 33-41.

Mai A.C., Vieira J.P. 2013. Review and consideration on habitat use, distribution and life history of Lycengraulis grossidens (Agassiz, 1829). Biota Neotrop. 13: 121-130.

Medeiros A.P.M. 2013. Distribuição e dieta da ictiofauna em área estuarino recifal da APA Barra do rio Mamanguape, Paraíba, Brasil. Graduation thesis, Univ. Fed. Paraiba, 87 pp.

Medeiros A.P.M., Xavier J.H.A., Rosa I.M.L. 2017. Diet and trophic organization of the fish assemblage from the Mamanguape River Estuary, Brazil. Lat. Am. J. Aquat. Res. 45: 879-890. https://doi.org/10.3856/vol45-issue5-fulltext-2

Mendoza-Carranza M. 2003. The feeding habits of gafftopsail catfish Bagre marinus (Ariidae) in Paraiso Coast, Tabasco, Mexico. Hidrobiológica 13: 119-126.

Menezes M.F. 1970. Alimentação da serra, Scomberomorus maculatus (Mitchill), em águas costeiras do Estado do Ceará. Arq. Ciênc. Mar 10: $171-176$.

Monteiro D.P., Giarrizzo T., Isaac V. 2009. Feeding ecology of juvenile dog snapper Lutjanus jocu (Bloch and Shneider, 1801) (Lutjanidae) in intertidal mangrove creeks in Curuçá estuary (Northern Brazil). Braz. Arch. of Biol. Tech. 52: 1421-1430. https://doi.org/10.1590/S1516-89132009000600014

Moraes R.L.G. 2012. Pesca, parasitismo e dieta alimentar da baúna Lutjanus alexandrei Moura \& Lindeman, 2007 nos ambientes costeiros do litoral sul de Pernambuco. Master thesis, Univ. Fed. Pernambuco, 90 pp.

Nelson J.S. 2006. Fishes of the World. New Jersey: John Wiley \& Sons. 601 pp.

Nizinski M.S., Munroe T.A. 2002. Order Clupeiformes, Engraulididae. In: Carpenter K.E (ed.), The Living Marine Resources of the Western Central Atlantic. FAO, Rome, pp.764-794.

Oliveira E., Favaro L. 2011. Reproductive biology of the flatfish Etropus crossotus (Pleuronectiformes: Paralichthyidae) in the Paranaguá Estuarine Complex, Paraná State, subtropical region of Brazil. Neotrop. Ichth. 9: 795-805.

Paiva A.C.G., Chaves P.D.T.D.C., Araújo M.E. 2008. Estrutura e organização trófica da ictiofauna de águas rasas em um estuário tropical. Rev. Bras. Zool. 25: 647-661. https://doi.org/10.1590/S0101-81752008000400010

Pimentel C.R. 2012. Organização trófica da comunidade de peixes de poças de maré da Praia dos Castelhanos (ES), Atlântico sudoeste tropical. Ph.D thesis, Univ. São Paulo, 82 pp.

Pina J.V. 2009. Avaliação da atividade reprodutiva da ictiofauna capturada na pesca artesanal de arrasto camaroeiro pela comunidade de Itapema do Norte, Itapoá, litoral norte de Santa Catarina. Master thesis, Univ. Fed. Paraná, 119 pp.

Pina J., Temóteo T.A.A., Sarmento G.C., et al. 2015. Hábito alimentar do Bairdiella ronchus (Cuvier, 1830) capturados em estuários de Pernambuco, nordeste do Brasil. XXI Enc. Bras. Ictiol., Pernambuco, Brazil.

Pombo M., Denadai M.R., Turra A. 2013. Seasonality, dietary overlap and the role of taxonomic resolution in the study of the diet of three congeneric fishes from a tropical bay. PLoS ONE 8: e56107. https://doi.org/10.1371/journal.pone.0056107

Possato F.E. 2010. Ecologia alimentar nas diferentes fases ontogenéticas de Cathorops spixii, C. agasiizii e Sciades herzbergii (Actinopterygii -Ariidae). Master thesis, Univ. Fed. Pernambuco, 89 pp.

Sampaio C.L.S., Nottingham M.C. 2008. Guia para identificação de peixes ornamentais -Volume 1: espécies marinhas. IBAMA, Brasília. $205 \mathrm{pp}$.

Santos F.B., Castro R.M.C. 2003. Activity, habitat utilization, feeding behaviour and diet of the sand moray Gymnothorax ocellatus (Anguilliformes, Muraenidae) in the South Western Atlantic. Biota Neotrop. 3: 1-7. https://doi.org/10.1590/S1676-06032003000100003

Schultz Y.D., Favaro L.F., Spach H.L. 2002. Aspectos reprodutivos de Sphoeroides greeleyi (Gilbert), Pisces, Osteichthyes, Tetraodontidae, da gamboa do Baguaçu, Baia de Paranaguá, Paraná, Brasil. Rev. Bras. Zool. 19: 65-76.

Schwamborn S.L.M. 2004. Dinâmica e organização trófica de assembleias de peixes associadas aos prados de capim marinho (Halodule wrightii) de Itamaracá, Pernambuco. Ph.D thesis, Univ. Fed. Pernambuco, 306 pp.

Segura-Berttolini E.C., Mendoza-Carranza M. 2013. Importance of male gafftopsail catfish, Bagre marinus (Pisces: Ariidae), in the reproductive process. Ciênc. Mar. 39: 29-39. https://doi.org/10.7773/cm.v39i1.2136

Shibuya A., Rosa R.S. 2011. Diet composition of Dasyatis marianae (Elasmobranchii: Dasyatidae) of Paraíba State, Brazil. Arq. Ciênc. Mar 44: 89-92.

Shinozaki-Mendes R., Santander-Neto J., Silva J., et al. 2013. Reproductive biology of Haemulon plumieri (Teleostei: Haemulidae) in Ceará state, Northeastern Brazil. Braz. J. Biol.73: 391-396. https://doi.org/10.1590/S1519-69842013000200020

Silva Junior C.B., Viana A.P., Lucena Frédou F., et al. 2015. Aspects of the reproductive biology and characterization of Sciaenidae captured as bycatch in the prawn trawling in the northeastern Brazil. Acta Scient. 37: 1-8. https://doi.org/10.4025/actascibiolsci.v37i1.24962

Silva C.M., Almeira Z. 2001. Alimentação de Rhizoprionodon Porosus (Elasmobranchii: Carcharhinidae) da Costa do Maranhão, Brasil. Bol. 
Inst. Pesca 27: 201-207

Silva J.T.O. Lopes P.R.D. 2002. Notas sobre a alimentação e morfologia do aparelho digestivo de Chloroscombrus chrysurus (Linnaeus, 1766) (Actinopterygii, Carangidae) na Praia de Ponta da Ilha (Ilha de Itaparica, Bahia). Rev. Bras. Zooc. 4: 179-192.

Sley A., Jarboui O., Ghorbel M., et al. 2009. Food and feeding habits of Caranx crysos from the Gulf of Gabes (Tunisia). J. Mar. Biol. Assoc. U.K. 89: 1377-1382.

Stefanoni M.F. 2008. Ictiofauna e ecologia trófica de peixes em ambientes praiais da ilha das peças, complexo estuarino de Paranaguá, Paraná. Master thesis, Univ. Fed. Paraná, 154 pp.

STRI. 2017. Evorthodus lyricus. Avaliable at biogeodb.stri.si.edu/caribbean/en/thefishes/species/4166

Teixeira R.L. 1994. Abundance, reproductive period, and feeding habits of eleotrid fishes in estuarine habitats of northeast Brazil. J. Fish Biol. 45: 749-761. https://doi.org/10.1111/j.1095-8649.1994.tb00941.x

Temóteo T.A.A. 2015. Caracterização do hábito alimentar das espécies do gênero Diapterus no complexo estuarina Itapissuma/Itamaracá. XV Jorn. Ens. Pesq. Ext. UFRPE, Pernambuco, Brazil.

Temóteo T.A.A., Pina J., Lira A.S., Sarmento G.C., et al. 2015. Alimentação de duas espécies do gênero Caranx capturados no litoral de Pernambuco. XXI Enc. Bras. Ictiol., Pernambuco, Brazil.

Trigueiro L.G.V. 2013. Dieta de Hyrpohamphus unifasciatus (Ranzani, 1841) (Actinopterygii: Hemiramphidae) no estuário do rio Mamanguape, Paraíba, Brasil. Graduation thesis, Univ. Est. Paraíba, 34 pp.

Vasconcellos M. 1998. Distribution patterns and feeding success of anchovy, Engraulis anchoita, larvae off southern Brazil. Sci. Mar. 62: 385-392. https://doi.org/10.3989/scimar.1998.62n4385

Vasconcelos Filho A.L. 1979. Estudo Ecológico da Região de Itamaracá, Pernambuco, Brasil. IV. Alimentação da Sardinha Bandeira, Opisthonema oglinum (Le Sueur, 1817), no Canal de Santa Cruz. Trop. Ocean. 14: 105-116.

Vasconcelos Filho A.L., Oliveira A.M.E. 1999. Composição e ecologia da ictiofauna do Canal de Santa Cruz (Itamaracá-PE, Brasil). Trab. Oceanog. UFPE 27: 101-113.

Vasconcelos Filho A.L., Neumann-Leitão S., Eskinazi-Leça E., et al. 2003. Trophic interactions between fish and other compartment communities in a tropical estuary in Brazil as indicator of environmental quality. Adv. Ecol. Sci. 18: 173-183.

Vasconcelos Filho A.L., Neumann-Leitão S., Eskinazi-Leça E., et al. 2009. Hábitos alimentares de consumidores primários da ictiofauna do sistema estuarino de Itamaracá (Pernambuco - Brasil). Rev. Bras. Eng. Pesca 4: 21-31.

Vasconcelos Filho A.L., Neumann-Leitão S., Eskinazi-Leca E., et al. 2010. Hábitos alimentares de peixes consumidores secundários do Canal de Santa Cruz, Pernambuco, Brasil. Trop. Oceanog. 38: 121-128.

Winik S., Carneiro M.H., Mendonça J.T. 2007. Alimentação da guaivira Oligoplites saliens (Bloch, 1793) (Perciformes: Carangidae) proveniente da pesca na região de Cananéia-SP. Inst. Pesca, São Paulo.

WoRMS Editorial Board. 2019. World Register of Marine Species. https://doi.org/10.14284/170

Wyanski D.M, Targett T.E. 2000. Development of transformation larvae and juveniles of Ctenogobius boleosoma, Ctenogobius shufeldti, and Gobionellus oceanicus (Pisces: Gobiidae) from western North Atlantic estuaries, with notes on early life history. Bull. Mar. Sci. 67: 709-728.

Yáñez-Arancibia A. 1986. Ecología de la zona costera. Análisis de siete tópicos. AGT: México, 189 pp.

Zahorcsak P., Silvano R.A., Sazima I. 2000. Feeding biologyof a guild of benthivorous fishes in a sandy shore on southeastern Brazilian coast. Rev. Bras. Biol. 60: 511-518. 